\title{
Pair and Impair, Even and Odd Form Fields, and Electromagnetism
}

\author{
Roldão da Rocha ${ }^{(1)}$ and Waldyr A. Rodrigues Jr. ${ }^{(2)}$ \\ ${ }^{(1)}$ Centro de Matemática, Computação e Cognição \\ Universidade Federal do ABC, 09210-170, Santo André, SP, Brazil \\ roldao.rocha@ufabc.edu.br \\ (2) Institute of Mathematics, Statistics and Scientific Computation \\ IMECC-UNICAMP CP 6065 \\ 13083-859 Campinas, SP, Brazil \\ walrod@ime.unicamp.br or walrod@mpc.com.br
}

\begin{abstract}
In this paper after reviewing the Schouten and de Rham definition of impair and pair differential form fields (not to be confused with differential form fields of even and odd grades) we prove that in a relativistic spacetime it is possible (despite claims in contrary) to coherently formulate electromagnetism (and we believe any other physical theory) using only pair form fields or, if one wishes, using pair and impair form fields together, in an appropriate way. Those two distinct descriptions involve only a mathematical choice and do not seem to lead to any observable physical consequence if due care is taken. Moreover, we show in details that a formulation of electromagnetic theory in the Clifford bundle formalism of differential forms where the two Maxwell equations of the so called free metric approach becomes a single equation is compatible with both formulations of electromagnetism just mentioned above. In addition we derive directly from Maxwell equation the density of force (coupling of the electromagnetic field with the charge current) that is a postulate in the free metric approach to electromagnetism. We recall also a formulation of the engineering version of Maxwell equations using electric and magnetic fields as objects of the same nature, i.e., without using polar and axial vectors.
\end{abstract}

\section{Introduction}

Cartan has popularized the use of differential forms which he apparently introduced in 1899 [4, and which are now indispensable tools in several mathematical 
and physical theories 1 . What is less known among physicists is that those objects come out in two versions, pair and impair differential forms (also called by some authors pseudo-forms or twisted forms)2, a concept which has its origin in the Heaviside formulation of electromagnetic theory in terms of polar and axial vector fields. Rigorously speaking, pair and impair forms are sections of different bundle 3 , but here to motivate our presentation we may say that pair forms living on an oriented spacetime are invariant under change of the coframe basis orientation (related to a fixed spacetime orientation) in which they are expressed — in particular, pair 0-forms are scalar functions - whereas impair forms change sign under change of the coframe basis orientation in which they are expressed, and in particular impair 0-forms are also known as pseudoscalar functions.

A definition 5 of such pair and impair differential forms has been originally introduced by de Rham [47 (but see also [58, 66) 6 and will be recalled below.

Of course, the theory of differential forms has been applied by many authors in the formulation of different physical theories (see, e.g., [54]), and in particular in electromagnetism. However, the formulations of that theory appearing, e.g., 3, 6, 9, 12, 19, 22, 31, 42, 61 make use only of pair differential form:7. It must be said that for those authors, the arena where charged particles and the electromagnetic field interact is a Lorentzian spacetime, that as well known is an oriented manifold8. On the other side authors like, e.g., [7, 11, 10, 13, 28, 26, 32, 34, 35, 43, 44, 45, 46, explicitly claim that impair forms are absolutely necessary for a consistent formulation of electromagnetism even in an oriented spacetime manifold and mainly if the spacetime is a bare manifold devoid of metric and affine structure9. Eventually, the main argument of the majority

\footnotetext{
${ }^{1}$ In particular, it seems that Cartan applied differential forms in the formulation of electromagnetism for the first time in [5].

${ }^{2}$ Impair forms are also called by some authors pseudo-forms or twisted forms.

${ }^{3}$ Pair forms are sections of the exterior algebra bundle $\bigwedge T^{*} M$ and impair forms are sections the bundle $\bigwedge_{-} T^{*} M \simeq L(M) \otimes \bigwedge^{*} M$ where $L(M)$ is a line bundle called the orientation bundle of $M$. Some details are given below.

${ }^{4}$ The orientation of a given coframe basis is not to be confused with the orientation of the manifold (part of the structure defining a spacetime) which is given by an arbitrary choice of a volume form. See below for details.

${ }^{5}$ We will give an alternative equivalent definition below.

${ }^{6}$ It must be said that that in de Rham's discussion of cohomology, impair forms have disappeared. In [49], this question is suitably studied by the investigation of the Grassmann and Clifford algebras over Peano spaces, introducing their respective associated extended algebras, and exploring these concepts also from the counterspace viewpoint. It was shown that the de Rham cochain, generated by the codifferential operator related to the regressive product, is composed by a sequence of exterior algebra homogeneous subspaces that are subsequently pair and impair.

${ }^{7}$ Some authors as e.g., 10, avoid the use of pair and impair forms by using instead (pair) multivector fields and pair forms.

${ }^{8}$ Almost all the authors in [3, 6, 9, 12, 19, 22, 31, 42, 61] even do not mention impair differential forms in their books and the few that mention those objects only say that they are necessary for a consistent integration theory on non oriented manifolds.

${ }^{9}$ The idea of developing electromagnetism using a manifold devoid of metric and affine structure is very old and appears in [8] and [36]. A complete set of references on the subject
} 
of those authors is that the current 3-form must be impair for otherwise its integral over an oriented 3 -chain (which gives the value of the charge in that region) does depend on the orientation chosen, a conclusion that those authors consider an absurd.

Moreover it must be said that the presentation of the differential equations of electromagnetism using the Clifford bundle formalism [54] uses only pair differential forms and, if the charge argument is indeed correct, it seems to imply that the Clifford bundle cannot be used to describe electromagnetism or any other physical theory. So, we must discuss in a thoughtful way the claims of 7. 11, 10, 13, 28, 26, 32, 34, 35, 43, 44, 45, 46, 63, and indeed, the main purpose of the present paper is to do that by showing that in a relativistic spacetime 10 the electromagnetic theory 11 can be rigorously presented with all fields involved being pair form fields. Of course, a presentation of electromagnetism in a oriented (even if bare) spacetime using appropriate pair and impair form fields is also correct, but as it will become clear below it seems to be nothing more than a simply option, not a necessity. Moreover, we show that contrary to a first expectation, the formulation of electromagnetism in the Clifford bundle [54] of (pair) form fields is automatically compatible with each one of those mentioned formulations of the theory, i.e., starting from Maxwell equations formulated as a single equation in the Clifford bundle, we can show that from that equation we can either obtain as a result of a straightforward mathematical choice two equations involving only pair forms or two equations such that one uses a pair form and the other impair forms.

This paper is organized as follows: in Section 2 we introduce the nature of the spacetime manifold used in the formulation of relativistic physical theories and recall Maxwell equations formulated with pair differential forms on Minkowski spacetime, calling the reader's attention to the fact that Maxwell equations describe only one aspect of electromagnetism, which is a theory describing the interaction of the electromagnetic field with charged particles (see specially Section 6). Moreover, we emphasize that although only the manifold structure of $M$ is enough for the writing of Maxwell equations, the remaining objects which defines the Minkowski spacetime structure play a fundamental role in the theory, as showed on several times in different sections of the paper. Section 3 is dedicated to the definition of the pair volume 4 -form and the pair Hodge star operator. Section 4 defines impair differential forms and in particular emphasizes the difference between the pair and the impair volume forms and the pair and impair Hodge star operators. Section 4 also discusses the fundamentals of electromagnetism in a medium and proves that, contrary to some claims [27, the recent discovery that the constitutive extensor of $\mathrm{Cr}_{2} \mathrm{O}_{3}$

up to 1960 is given in 64. Such an approach to electromagnetism has also been used in [57] and now is advocated by many authors, see specially [28] (and of course, the arXiv) for modern references.

\footnotetext{
${ }^{10}$ The concept of a relativistic spacetime as used in this paper is recalled in Section 2 and Subsection 3.2 .

${ }^{11}$ This includes even the case of regions involving a non dispersive medium that can be described by effective Lorentzian spacetimes [43].
} 
has a term proportional to the Levi-Civita symbol in no way implies that this discovery is the proof that impair forms must be used in the formulation of electromagnetism. Section 5 recalls the Clifford bundle formulation of Maxwell equation 12 , proving as already mentioned that it is compatible with those two formulations (only pair and pair and impair) of that equations. Section 6 shows how the force density that is postulated in the presentation of electromagnetism in 28] is directly contained in Maxwell equation. Moreover we show in Section 6 that the equation (which contains the force density) describing the interaction of the charged particles with the field automatically knocks down the charge argument mentioned above. In Section 7 using the Pauli algebra bundle we present the engineering formulation of electromagnetism in terms of the electric and magnetic fields $\mathbf{E}$ and $\mathbf{B}$ and emphasize that in this formulation which necessarily needs a choice of a volume element we do not need to introduce the so called axial vector fields and moreover that the circulation of the magnetic field around a (very long) wire conducting current is conventional. Finally in Section 8 we present our concluding remarks.

\section{Nature of the Spacetime Manifold and of the Electromagnetic Field.}

Every physical theory starts by modeling the arena (spacetime) where physical phenomena are supposed to happen. It is a well known fact that when gravitation can be neglected, the motion (classical or quantum) of particles and fields occurs in an arena which is modeled by Minkowski spacetime, i.e., a structure $\left(M, \boldsymbol{g}, D, \tau_{g}, \uparrow\right)$, where $M$ is a 4-dimensional manifold diffeomorphic to $\mathbb{R}^{4}$, $\mathbf{g} \in \sec T_{0}^{2} M$ is a Lorentzian metric, $D$ is the Levi-Civita connection of $\mathbf{g}$ (i.e., $\mathbf{T}(D)=0$, where $\mathbf{T}$ is the torsion tensor associated to the connection $D), \mathbf{R}(D)$ is the curvature tensor associated with $D, \tau_{\mathrm{g}} \in \sec \bigwedge^{4} T^{*} M$ is the metric volume element, i.e., a pain 13 -form defining a spacetime orientation and $\uparrow$ denotes time orientation 14 .

Classical electromagnetism according to Feynman is the theory which describes the interaction of objects called charged particles and the electromagnetic field $F \in \sec \bigwedge^{2} T^{*} M$ called field strength. For the purposes of this paper a charged particle is described by a triple $(m, q, \sigma)$, where (conventionally) $m \in \mathbb{R}^{+}$is the mass parameter and based on experimental facts $q$ (the charge) is a non null integral multiple of an elementary charge denoted $|e|$. It is extremely important to keep in mind for the objectives of the present paper that the sign of $q$ to be attributed to any charge depends on a convention, which will be scrutinized latter (Remark 17 and Section 4.3). Moreover, $\sigma: \mathbb{R} \rightarrow \mathbb{M}$ is timelike curve pointing to the future 15 . We parametrize $\sigma$ in such a way that

\footnotetext{
${ }^{12}$ No misprint here. To know why look at Eq.(52).

${ }^{13}$ See below for the definition of pair and impair forms.

${ }^{14}$ More details may be found, e.g. in [54, 56].

${ }^{15}$ This being the reason why we suppose that spacetime is time orientable.
} 
$\mathbf{g}\left(\sigma_{*}, \sigma_{*}\right)=1$ and define a 1 -form field over $\sigma$, denoted by $v=\mathbf{g}\left(\sigma_{*}\right)$. Given a finite collection of particles $\left(m^{(i)}, q^{(i)}, \sigma^{(i)}\right), i=1,2, \ldots, n$, we define the current for the $i$-particle as the 1 -form field $J^{(i)}=q^{(i)} v^{(i)}$ over $\sigma$. The total current of the system is given by

$$
J=\sum_{i} J^{(i)}
$$

which support are the set of timelike lines $\cup_{i} \sigma^{(i)}$. If we introduce a global coordinate chart for $M$ with coordinates $\left\{\mathrm{x}^{\mu}\right\}$ in the Einstein-Lorentz-Poincaré gauge 16 then we can write

$$
\begin{gathered}
J=\sum_{i} J_{\mu}^{(i)} \gamma^{\mu}, \\
J_{\mu}^{(i)}=\eta_{\mu \nu} q^{(i)} \int \delta^{(4)}\left(\mathrm{x}^{\beta}-\mathrm{x}^{\beta} \circ \sigma^{(i)}\left(s_{(i)}\right)\right) \frac{d \mathrm{x}^{\nu} \circ \sigma^{(i)}\left(s_{(i)}\right)}{d s_{(i)}} d s_{(i)},
\end{gathered}
$$

with $s_{(i)}$ being the proper time along $\sigma^{(i)}$. Before going on we must say that if the density of particles is very large we may eventually approximate $J$ by a continuous section of $\bigwedge^{1} T^{*} M$ or at least by a de Rham current 47. It is an empirical fact that $F$ is closed, i.e., $d F=0$ and moreover $\sqrt{17}, \mathbf{J}=\star \underset{g}{*} J \in$ sec $\bigwedge^{3} T^{*} M$ is exact, i.e., $\mathbf{J}=-d G$ for $G \in \sec \bigwedge^{3} T^{*} M$, called the excitation field. Those empirical observations are written as

$$
d F=0, d G=-\mathbf{J}
$$

and known as Maxwell equations.

Remark 1 Before we proceed it must be said that if we forget the fact that the carriers of charges are particles and simply suppose that experimentally all we have is a $\mathbf{J} \in \sec \bigwedge^{3} T^{*} M$ that is conserved (i.e., $d \mathbf{J}=\mathbf{0}$ ), then supposing that the manifold where $\mathbf{J}$ lives is star-shaped, we have $d G=-\mathbf{J}$. Using moreover the fact that $d F=0$ (meaning that magnetic monopoles do not exist) it is a mathematical fact that the system of differential equations given by Eq.(4) does need for its writing only the structure of the bare manifold structure $M$, i.e., it does not need the additional objects $\left(\mathrm{g}, D, \tau_{\mathrm{g}}, \uparrow\right)$ entering the structure of Minkowski spacetime18. However, electromagnetism is not only Maxwell equations, we must yet to specify the way that two currents $\mathbf{J}^{(1)}, \mathbf{J}^{(2)} \sec \bigwedge^{3} T^{*} M$ interact. And to do this we shall need to use the additional structure, as we shall see.

\footnotetext{
${ }^{16}$ Given a Minkowski spacetime structure, global coordinates $\left\{\mathrm{x}^{\mu}\right\}$ for $M \simeq \mathbb{R}^{4}$ are said to be in the Einstein-Lorentz-Poincaré gauge $(E L P G)$ if and only if the following conditions hold: $g=\eta_{\mu \nu} d \mathrm{x}^{\mu} \otimes d \mathrm{x}^{\nu}, D \frac{\partial}{\partial \mathrm{x}^{\mu}} \frac{\partial}{\partial \mathrm{x}^{\nu}}=0$. Of course, as well known, there exists an infinity of coordinate functions related by Poincaré transformations satisfying these conditions. We shall write in what follows $\gamma^{\mu}:=d \mathrm{x}^{\mu}$ and $\gamma_{\mu}=\eta_{\mu \nu} \gamma^{\nu}$.

${ }^{17}$ The symbol $\star$ means the pair Hodge dual operator, and its definition is given below.

${ }^{18}$ This has been originally observed by Cartan in [5].
} 
To proceed with our presentation of electromagnetism we must recall that as it is well known the metric tensor can be used to give a Clifford bundle structure to $\bigwedge T^{*} M=\bigoplus_{p=0}^{4} \bigwedge^{p} T^{*} M$, which will be called (for reasons to be explained below) the pair bundle of differential forms. The Clifford bundle of nonhomogeneous differential forms is denoted by $19 \mathcal{C} \ell(M, g)$, where $g \in \sec T_{2}^{0} M$ denotes the metric of the cotangent bundle, such that for any arbitrary basis $\left\{e_{\mu}\right\}$ of $T U \subseteq T M$ and dual basis $\left\{\boldsymbol{\theta}^{\mu}\right\}$ of $T^{*} U \subseteq T^{*} M$ (U are open sets in $M), \theta^{\mu} \in \sec \bigwedge^{1} T^{*} U \subseteq \sec \bigwedge^{1} T^{*} M \hookrightarrow \sec \mathcal{C} \ell(M, g)$, we have $\boldsymbol{g}=g_{\mu \nu} \theta^{\mu} \otimes \theta^{\nu}$, $g=g^{\mu \nu} e_{\mu} \otimes e_{\nu}$ and $g_{\mu \nu} g^{\nu \lambda}=\delta_{\mu}^{\lambda}$.

Remark 2 We recall that any section of $\bigwedge^{r} T^{*} M$ is said to be a r-graded form field (or r-form for short). Sometimes it is said to be of even or odd grade, depending on whether $r$ is even or odd. This classification is not to be confused to the concept of de Rham pair and impair forms, to be introduced below.

\subsection{Energy-Momentum 1-Form and Energy-Momentum Tensor for the System of Charged Particles}

For use in Section 6 we define now the energy-momentum 1-form for a charged particle $\left(m^{(i)}, q^{(i)}, \sigma^{(i)}\right)$ as the 1 -form field $p^{(i)}$ over $\sigma^{(i)}$ given by

$$
p^{(i)}=m^{(i)} v^{(i)}
$$

and it is obvious that $p^{(i)} \cdot p^{(i)}=\left(m^{(i)}\right)^{2}$. In an inertial frame $\mathbf{I}=\frac{\partial}{\partial \mathrm{x}^{0}}$ associated to the coordinates $\left\{\mathrm{x}^{\mu}\right\}$ for $M$ in ELPG at time $\mathrm{x}^{0}=t$, the particles will occupy different spacetime points $\left(t, \mathrm{x}_{(i)}^{1}(t), \mathrm{x}_{(i)}^{2}(t), \mathrm{x}_{(i)}^{3}(t)\right)$. We can define the total momentum of the particles at time $t$ only if it is licit to sum distinct 1 -forms at different tangent spaces of $M$. This, of course requires an absolute parallelism and here is then a place where the flat connection $D$ that was introduced in the structure of Minkowski spacetime becomes necessary. It permits us to write the total momentum of the particles at time $t$ as

$$
P(t)=\sum_{i} p^{(i)}(t)
$$

a necessary concept needed in order to be possible to talk about energy-momentum conservation for the system of particles and the electromagnetic field (see Section 6). Besides the momentum 1-form of the particles we shall need also to introduce the energy-momentum 1-forms $\mathbf{T}^{\alpha} \in \sec \bigwedge^{1} T^{*} M$ for the system of charged particles. We have:

\footnotetext{
${ }^{19}$ Details on the construction of $\mathcal{C} \ell(M, g)$ may be found, e.g., in [54.

${ }^{20} \mathrm{An}$ inertial frame is defined as a time like vector field $\mathbf{I}$ such that $\boldsymbol{g}(\mathbf{I}, \mathbf{I})=1$ and $D \mathbf{I}=\mathbf{0}$. More details if need may be found, e.g., at 54.
} 


$$
\begin{aligned}
\mathbf{T}^{\alpha} & =\mathbf{T}^{\alpha \beta} \gamma_{\beta}, \\
\mathbf{T}^{\alpha \beta} & =\sum_{i} \eta^{\alpha \mu} \int p_{\mu}^{(i)}(s) \frac{d}{d s} \mathbf{x}^{\beta} \circ \sigma^{(i)}\left(s_{(i)}\right) \delta^{4}\left(\mathrm{x}^{\kappa}-\mathrm{x}^{\kappa} \circ \sigma^{(i)}\left(s_{(i)}\right)\right) d s_{(i)} .
\end{aligned}
$$

\section{The Pair Metric Volume Element $\tau_{g}$}

First introduce an arbitrary $\mathbf{g}$-orthonormal basis $\left\{\mathbf{e}_{\alpha}\right\}$ for $T M$ and corresponding dual basis $\left\{\boldsymbol{\theta}^{\alpha}\right\}$ for $T^{*} M$. Then, $\boldsymbol{g}\left(\mathbf{e}_{\alpha}, \mathbf{e}_{\beta}\right)=\eta_{\alpha \beta}$ and $g\left(\boldsymbol{\theta}^{\alpha}, \boldsymbol{\theta}^{\beta}\right)=\boldsymbol{\theta}^{\alpha} \cdot \boldsymbol{\theta}^{\beta}=$ $\eta^{\alpha \beta}$ and $\boldsymbol{\theta}^{\alpha}\left(\mathbf{e}_{\beta}\right)=\delta_{\beta}^{\alpha}$, where the matrix with entries $\eta_{\alpha \beta}$ and the matrix with entries $\eta^{\mu \nu}$ are equal to the diagonal matrix $\operatorname{diag}(1,-1,-1,-1)$. We define a pair metric volume21 $\tau_{g} \in \sec \bigwedge^{4} T^{*} M$ by

$$
\tau_{\mathrm{g}}:=\boldsymbol{\theta}^{0} \wedge \boldsymbol{\theta}^{1} \wedge \boldsymbol{\theta}^{2} \wedge \boldsymbol{\theta}^{3}
$$

Remark 1 An orientation for $M$ as we already said above is a free choice of an arbitrary volume element 22 Before proceeding let us introduce arbitrary coordinates $\left\{x^{\mu}\right\}$ for $U \subset M$ and $\left\{x^{\prime \mu}\right\}$ for $U^{\prime} \subset M, U \cap U^{\prime} \neq \varnothing$ such that

$$
\boldsymbol{\theta}^{\alpha}=h_{\mu}^{\alpha} d x^{\mu}, \boldsymbol{\theta}^{\alpha}=h_{\mu}^{\prime \alpha} d x^{\prime \mu}
$$

Let $\mathbf{h}$ and $\mathbf{h}^{\prime}$ the matrices with entries $h_{\mu}^{\alpha}$ and $h_{\mu}^{\prime \alpha}$. Then, e.g.,

$$
\operatorname{det}\left(g_{\alpha \beta}\right)=(\operatorname{det} \mathbf{h})^{2} \operatorname{det}\left(\eta_{\alpha \beta}\right),
$$

and

$$
\sqrt{\left|\operatorname{det}\left(g_{\alpha \beta}\right)\right|}=|\operatorname{det} \mathbf{h}| \sqrt{\left|\operatorname{det}\left(\eta_{\alpha \beta}\right)\right|}=|\operatorname{det} \mathbf{h}|
$$

The expression of $\tau_{g}$ in the bases $\left\{d x^{\mu}\right\}$ and $\left\{d x^{\prime \mu}\right\}$ are respectively

$$
\begin{aligned}
\tau_{g} & =\frac{1}{4 !} \tau_{i_{0} \ldots i_{3}} d x^{i_{0}} \wedge \cdots \wedge d x^{i_{3}}=\tau_{0123} d x^{0} \wedge \cdots \wedge d x^{3} \\
& =\frac{\operatorname{det} \mathbf{h}}{|\operatorname{det} \mathbf{h}|} \sqrt{\left|\operatorname{det}\left(g_{\alpha \beta}\right)\right|} d x^{0} \wedge \cdots \wedge d x^{3},
\end{aligned}
$$

and

$$
\tau_{g}=\frac{1}{4 !} \tau_{j_{0} \ldots j_{3}}^{\prime} d x^{\prime j_{0}} \wedge \cdots \wedge d x^{\prime j_{3}}=\tau_{0123}^{\prime} d x^{\prime 0} \wedge \cdots \wedge d x^{\prime 3} .
$$

Now writing $\Lambda_{j_{p}}^{i_{p}}=\frac{\partial x^{i_{p}}}{\partial x^{\prime j p}}, \operatorname{det} \Lambda=\operatorname{det}\left(\frac{\partial x^{i}}{\partial x^{\prime j}}\right)$ we have (remember that $\tau_{i_{0} \ldots i_{3}}=$ $\left.\varepsilon_{i_{0} \ldots i_{3}}^{0123} \tau_{0123}=\varepsilon_{i_{0} \ldots i_{3}}^{0123} \frac{\operatorname{det} \mathbf{h}}{\operatorname{det} \mathbf{h} \mid} \sqrt{\left|\operatorname{det}\left(g_{i j}\right)\right|}\right)$

$$
\tau_{j_{0} \ldots j_{3}}^{\prime}=\Lambda_{j_{0}}^{i_{0}} \ldots \Lambda_{j_{3}}^{i_{3}} \tau_{i_{0} \ldots i_{3}} .
$$

\footnotetext{
${ }^{21}$ The impair volume elements is defined in Section 4.1.

${ }^{22}$ Of course, an arbitrary manifold $M$, even if orientable, when equipped with an arbitrary Lorentzian metric field $g$ does not in general admit a global g-orthonormal cotetrad field, so, in this case the introduction of $\tau_{\mathrm{g}}$ is a little more complicated [6, 9]. However, all manifolds $M$ part of a Lorentzian spacetime structure that admits spinor fields have a global g-orthonormal cotetrad field. This is a result from a famous theorem due to Geroch [21].
} 
Also, since $\sqrt{\left|\operatorname{det}\left(g_{i j}^{\prime}\right)\right|}=|\operatorname{det} \Lambda| \sqrt{\left|\operatorname{det}\left(g_{i j}\right)\right|}$, we end with

$$
\begin{aligned}
\tau_{0123}^{\prime} & =\operatorname{det} \Lambda \tau_{0123}:=\Delta^{-1} \tau_{0123} \\
& =\frac{\operatorname{det} \mathbf{h}}{|\operatorname{det} \mathbf{h}|} \frac{\operatorname{det} \Lambda}{|\operatorname{det} \Lambda|} \sqrt{\left|\operatorname{det}\left(g_{i j}^{\prime}\right)\right|} .
\end{aligned}
$$

Remark 3 We want to emphasize here that with the choice $\frac{\operatorname{det} \mathbf{h}}{|\operatorname{det} \mathbf{h}|}=+1$, the coordinate expression for $\tau_{g}$ in the basis $\left\{d x^{\mu}\right\}$ becomes the one appearing in almost all textbooks, i.e., $\sqrt{\left|\operatorname{det}\left(g_{i j}\right)\right|} d x^{0} \wedge \cdots \wedge d x^{3}$. However the coordinate expression for $\tau_{\mathrm{g}}$ in the basis $\left\{d x^{\prime \mu}\right\}$ is $\sqrt{\left|\operatorname{det}\left(g_{i j}^{\prime}\right)\right|} d x^{\prime 0} \wedge \cdots \wedge d x^{\prime 3}$ only if $\frac{\operatorname{det} \Lambda}{|\operatorname{det} \Lambda|}=$ +1 . The omission of the factor $\frac{\operatorname{det} \Lambda}{|\operatorname{det} \Lambda|}$ in the textbook presentation of $\tau_{\mathrm{g}}$ is the source of a big confusion and eventually responsible for a statement saying that the volume element must be an impair 4-form. An impair volume element is an object different from $\tau_{g}$ and will be introduced in Section 4.1.

Comparing Eq.(15) to Eq.(8.1) of Schouten's book [58] we see the reason why a quantity that "transforms" like in Eq.(15) is called a scalar- $\Delta$-density of weight 1. Despite being fan of Schouten's book, the authors think that such a nomenclature may induce confusion, unless expressed in a coordinate free way as, e.g., done in [1, 2, 38.

\subsection{The Pair Hodge Star Operator}

A pair metric volume element $\tau_{g}$ permits us to define an isomorphism between $\bigwedge^{p} T^{*} M \hookrightarrow \mathcal{C} \ell(M, g)$ and $\bigwedge^{4-p} T^{*} M \hookrightarrow \mathcal{C} \ell(M, g)$, given by

$$
\begin{aligned}
\underset{\tau_{g}}{\star}: \bigwedge^{p} T^{*} M & \rightarrow \bigwedge^{4-p} T^{*} M \\
A_{p} & \mapsto \underset{\tau_{g}}{\star} A_{p}:=\tilde{A}_{p} \tau_{g}
\end{aligned}
$$

In Eq.(17) $\tilde{A}_{p} \tau_{\mathrm{g}}$ means the Clifford product between the Clifford fields $\tilde{A}_{p}$ and $\tau_{g}$, and $\tilde{A}_{p}$ is the reverse 23 of $A_{p}$. Let $\left\{\mathrm{x}^{\mu}\right\}$ be global coordinates in the ELPG and $\left\{\gamma^{\mu}=d \mathrm{x}^{\mu}\right\}$ an orthonormal cobasis, i.e., $g\left(\gamma^{\mu}, \gamma^{\nu}\right):=\gamma^{\mu} \cdot \gamma^{\nu}=\eta^{\mu \nu}$.

In this case we can write $\tau_{g}=\gamma^{5}=\gamma^{0} \wedge \gamma^{1} \wedge \gamma^{2} \wedge \gamma^{3}=\gamma^{0} \gamma^{1} \gamma^{2} \gamma^{3}$ and the calculation of the action of the Hodge dual operator on a $p$-form becomes an elementary algebraic operation 24. We also suppose that $\tau_{g}=\gamma^{5}$ defines a positive orientation (also called right handed orientation), and it is trivial to verify that 25

$$
\tau_{g} \tau_{g}=\tau_{g} \cdot \tau_{g}=\left(\gamma^{5}\right)^{2}=-1
$$

\footnotetext{
${ }^{23}$ See, e.g., 54 for details.

${ }^{24}$ Of course, our statement is true only for someone that knows a little bit of Clifford algebra, as it is supposed to be the case of a reader of the present article.

${ }^{25}$ The Clifford product in this paper is represented by juxtaposition of symbols following the convention of [54].
} 
Before we proceed, recall that we can show trivially that the definition given by Eq.(17) is equivalent to the standard one, i.e., for any $A_{p}, B_{p} \in \sec \bigwedge^{p} T^{*} M$, it follows that

$$
B_{p} \wedge \underset{\tau_{\mathrm{g}}}{\star} A_{p}=\left(B_{p} \cdot A_{p}\right) \tau_{\mathrm{g}}
$$

Remark 4 As defined, the object $A_{p}^{\prime}=\underset{\tau_{g}}{\star} A_{p}$ is a legitimate pair form, although it depends, as it is obvious from Eq.(17), of the chosen orientation $\tau_{g}$. Some authors (like e.g., 28]) assert that the Hodge star operator maps a pair form into an impair one (see the definition of impair forms below). What does this statement mean given our definition that the (pair) Hodge star operator changes an even grade form to an odd grade form (and vice versa)? It means that an impair Hodge operator that maps a pair form into an impair one can also be defined and of course is a concept different from the one just introduced. The impair Hodge operator will be presented and discussed in Section 4.1. To avoid any possible confusion on this issue, let us bethink that there may two different Hodge operators associated with the same metric $\mathbf{g}$. Indeed, given the metric $\mathbf{g}$ and a (pair) metric volume 4 -form $\tau_{g}^{\prime} \neq \tau_{g}$ with $\tau_{g}^{\prime 2}=-1$ we may define another Hodge star operator

$$
\begin{aligned}
\underset{\tau_{g}^{\prime}}{\star}: \bigwedge^{p} T^{*} M & \rightarrow \bigwedge^{4-p} T^{*} M \\
A_{p} & \mapsto \underset{\tau_{g}^{\prime}}{\star} A_{p}:=\tilde{A}_{p} \tau_{g}^{\prime}
\end{aligned}
$$

Now, there are only two possibilities for $\tau_{g}^{\prime}$. Either $\tau_{g}^{\prime}=\tau_{g}$ or $\tau_{g}^{\prime}=-\tau_{g}$. In the second case we say that $\tau_{\mathrm{g}}^{\prime}$ defines a negative or left handed orientation. It is obvious that in this case we have

$$
\underset{\tau_{g}^{\prime}}{\star} A_{p}:=-\underset{\tau_{g}}{\star} A_{p}
$$

but we insist: both $\underset{\tau_{g}^{\prime}}{\star} A_{p}$ and $\underset{\tau_{g}}{\star} A_{p}$ are legitimate pair 4-forms.

Following Feynman, we take the view that only the field $F$ is fundamental and that the charge carriers moves in the vacuum (Lorentz vacuum). It is then necessary to find the relation between $G$ and $F$ for the vacuum. It is an empirical fact that once a spacetime orientation $\tau_{g}$ is fixed (by arbitrary choice) we get a correct description of electromagnetic phenomena in vacuum 26 by taking

$$
G:=\underset{\tau_{g}}{\star} F
$$

\footnotetext{
${ }^{26}$ Of course, any formulation of electrodynamics in a medium must take as true the equations in vacuum and the properties of matter which are supposed to be described (due to the obvious difficulties with the many body problem) by an approximate phenomenological theory derived, e.g., from quantum mechanics. This is the point of view of Feynman [17] which we endorse.
} 
Remark 5 Until now, we have only used pair forms in our formulation of electromagnetism, but we call the reader's attention to the fact that if we choose the opposite spacetime orientation $\tau_{g}^{\prime}$ and $\underset{\tau_{g}^{\prime}}{\star}$, we must put

$$
G=-\underset{\tau_{g}^{\prime}}{\star} F
$$

if we want to preserve the non homogeneous Maxwell equation $d G=-\mathbf{J}$.

\section{Impair Forms}

The definition of the Hodge dual leaves it clear that different orientations (i.e., different pair volume element forms differing by a sign) produce duals - in the Hodge's sense - differing by a sign. This elementary fact is sometimes confused with the concept of impair forms introduced by de Rham [47]. From a historical point of view it must be recalled that de Rham pair and impair forms are only

a modern reformulation of objects already introduced by Weyl [66] and then by Schouten 58.

Let $\left\{e_{\mu}\right\}$ and $\left\{e_{\mu}^{\prime}\right\}$ be arbitrary bases for sections of $T U \subset T M$ and $T U^{\prime} \subset$ $T M\left(U^{\prime} \cap U \neq \varnothing\right)$ and $\left\{\theta^{\mu}\right\}$ and $\left\{\theta^{\prime \mu}\right\}$ be respectively bases for sec $\bigwedge T^{*} U \subset$ $\sec \bigwedge T^{*} M \hookrightarrow \sec \mathcal{C} \ell(M, g)$ and $\sec \bigwedge T^{*} U^{\prime} \subset \sec \bigwedge T^{*} M \hookrightarrow \sec \mathcal{C} \ell(M, g)$ which are respectively dual to the bases $\left\{e_{\mu}\right\}$ and $\left\{e_{\mu}^{\prime}\right\}$. Let $\omega=\frac{1}{4 !} \omega_{i_{0} \ldots i_{3}} d x^{i_{0}} \wedge \cdots \wedge d x^{i_{3}}$ and $\omega^{\prime}=\frac{1}{4 !} \omega_{i_{0} \ldots i_{3}}^{\prime} d x^{\prime i_{0}} \wedge \cdots \wedge d x^{\prime i_{3}}$ and let $\tau_{\mathrm{g}}$ be the orientation of the spacetime, which we recall is a free choice.

Definition 6 We say that the ordered coframe basis $\left\{\theta^{\mu}\right\},\left\{\theta^{\prime \mu}\right\}$ (or simply $\left.\omega, \omega^{\prime}\right)$ are positive or right-handed oriented relative to $\tau_{\mathrm{g}}$ if

$$
o(\omega):=-\omega \cdot \tau_{g}>0, o\left(\omega^{\prime}\right)=-\omega^{\prime} \cdot \tau_{g}>0,
$$

and if

$$
o(\omega):=-\omega \cdot \tau_{g}<0, o\left(\omega^{\prime}\right)=-\omega^{\prime} \cdot \tau_{g}<0 .
$$

the bases are said to be negative or left-handed oriented.

Remark 7 It is very important not to confuse the concept of orientation of a coframe basis given by o $(\omega)$ with the spacetime orientation given by $\tau_{g}$. But of course, the orientation of a coframe changes if it is referred to another volume element with different orientation.

Remark 8 Also, suppose that a given manifold $M$ is non orientable. In this case we define the relative orientation of the basis $\left\{\theta^{\mu}\right\},\left\{\theta^{\prime \mu}\right\}$ on $U \cap U^{\prime}$ by saying that they have the same orientation if $\omega \cdot \omega^{\prime}>0$ and opposite orientation if $\omega \cdot \omega^{\prime}<0$. In the following the symbol $o(\omega)$ will be used according to Definition [6 if we are referring to an orientable manifold. In the eventual case where we referred to a non oriented manifold that even does not carry a metric field, o( $\left.\omega^{\prime}\right)$ will mean the relative orientation of a given basis $\left\{\theta^{\prime \mu}\right\}$ in $U \cap U^{\prime}$ relative to $\left\{\theta^{\mu}\right\}$, given by sign of Jacobian determinant $\operatorname{det} \Lambda /|\operatorname{det} \Lambda|$. 
Definition 9 An impair p-form field $\stackrel{\Delta}{A_{p}}$ is an equivalence class of pairs $\left(\triangle_{p}^{\omega}, o(\omega)\right)$, where $\stackrel{\triangle}{A}_{p}^{\omega} \in \sec \bigwedge^{p} T^{*} M$ a pair form-called the representative of $\vec{A}_{p}$ on the basis $\left\{\theta^{\mu}\right\}-$ is given by

$$
\stackrel{\triangle}{A}_{p}^{\omega}=o(\omega) \frac{1}{p !} \stackrel{\triangle}{A}_{i_{1} \ldots i_{p}} \theta^{i_{1}} \wedge \cdots \wedge \theta^{i_{p}}
$$

Given the pairs $\left(\stackrel{\Delta}{A}_{p}^{\omega}, o(\omega)\right)$ and $\left(\stackrel{\Delta}{A}_{p}^{\omega^{\prime}}, o\left(\omega^{\prime}\right)\right)$ where $\stackrel{\stackrel{\Delta}{A}_{p}^{\prime}}{\omega^{\prime}} \in \sec \bigwedge^{p} T^{*} M$ is given by

$$
{\stackrel{\triangle}{A_{p}}}_{\omega^{\prime}}=o\left(\omega^{\prime}\right) \frac{1}{p !} \stackrel{\triangle}{A}_{j_{1} \ldots i_{p}} \theta^{j_{1}} \wedge \cdots \wedge \theta^{j_{p}}
$$

we say that they are equivalent if one of the two cases holds:

$$
\begin{aligned}
& \text { (a) if } o(\omega)=o\left(\omega^{\prime}\right), \quad \stackrel{\triangle}{A}_{p}^{\omega}=\stackrel{\Delta}{A}_{p}^{\omega^{\prime}} \\
& \text { (b) if } o(\omega)=-o\left(\omega^{\prime}\right), \quad \stackrel{\Delta}{A}_{p}^{\omega}=-\triangle_{p}^{\omega^{\prime}}
\end{aligned}
$$

Remark 10 Let $\left\{e_{\mu}=\frac{\partial}{\partial \mathrm{x}^{\mu}}\right\}$ and $\left\{e_{\mu}^{\prime}=\frac{\partial}{\partial x^{\prime} \mu}\right\}$ be coordinate bases where $\left\{\mathrm{x}^{\mu}\right\}$ are global coordinates in the Einstein Lorentz-Poincaré gauge for $U \subset M$ and $\left\{x^{\mu}\right\}$ coordinates for $U^{\prime} \subset M$. Now the orientation of $\left\{\gamma^{\mu}=d \mathrm{x}^{\mu}\right\}$ being taken as positive, if we simply write (as did de Rham)

$$
\begin{aligned}
& \stackrel{\triangle}{A}_{p}^{\omega}=\frac{1}{p !} \stackrel{A}{A}_{i_{1} \ldots i_{p}} d \mathrm{x}^{i_{1}} \wedge \cdots \wedge d \mathrm{x}^{i_{p}}, \\
& \stackrel{\triangle}{A}_{p}^{\omega^{\prime}}=\frac{1}{p !} \stackrel{\leftrightarrow}{A}_{j_{1} \ldots j_{p}}^{\prime} d x^{\prime j_{1}} \wedge \cdots \wedge d x^{\prime j_{p}},
\end{aligned}
$$

then we must have

$$
\hat{A}_{j_{1} \ldots j_{p}}^{\prime}=\frac{\operatorname{det} \Lambda}{|\operatorname{det} \Lambda|} \Lambda_{j_{1}}^{i_{1}} \ldots \Lambda_{j_{p}}^{i_{p}} \hat{A}_{i_{1} . . i_{p}}
$$

with $\operatorname{det} \Lambda=\operatorname{det}\left(\frac{\partial x^{i}}{\partial x^{\prime j}}\right)$. Eq. (31) is the definition of an impair form given by de Rham [47] (see also [58]).

Remark 11 It is very important to note that, since according to Definition 6 an impair p-form is an equivalence class of pairs $\left(\Delta_{p}^{\omega}, o(\omega)\right)$ where each $\stackrel{\Delta}{A}_{p}$ is a pair $p$-form and $o(\omega)$ denotes the basis orientation, Recall that if spacetime is oriented and we define $o(\omega)$ by Eqs.(24) and (25), then it depends on the spacetime orientation $\tau_{g}$, and it follows that each pair p-form representative of an impair p-form depends also on the choice of the spacetime orientation. Indeed, if we change the spacetime orientation from $\tau_{\mathrm{g}}$ to $\tau_{g}^{\prime}=-\tau_{\mathrm{g}}$ the orientation of the coframe $\left\{\theta^{\mu}\right\}$ changes to $o^{\prime}(\omega)=-\omega \cdot \tau_{\mathrm{g}}^{\prime}=-o(\omega)$. 
We denote the bundle of impair $p$-forms by $\bigwedge_{-}^{p} T^{*} M$ and the exterior bundle $\bigwedge_{-} T^{*} M=\bigoplus_{p=0}^{4} \bigwedge_{-}^{p} T^{*} M$. Let $\stackrel{\triangle}{A}_{p} \in \sec \bigwedge_{-}^{p} T^{*} M$ denote that the impair $p$-form field $\stackrel{\triangle}{A}_{p}$ is a section of $\bigwedge_{-}^{p} T^{*} M$

Remark 12 We can easily show that $\bigwedge T^{*} M$ as defined above is isomorphic to $L(M) \otimes \bigwedge T^{*} M$ (whose sections are line-bundle-valued multiforms on $\left.M\right)$ We write [1, 2]

$$
\bigwedge_{-} T^{*} M \simeq L(M) \otimes \bigwedge T^{*} M
$$

where $L(M)$ is the so called orientation line bundle of $M$, a vector bundle with typical fiber $\mathbb{R}$ and where the transition functions are defined as follows. Let $\left\{\left(U_{\alpha}, \varphi_{\alpha}\right)\right\}$ be a coordinate covering of $M$ with transition functions given by $t_{\alpha \beta}=$ $\varphi_{\alpha} \circ \varphi_{\beta}^{-1}$. Then, the transition functions of $L(M)$ are given by $J\left(t_{\alpha \beta}\right) /\left|J\left(t_{\alpha \beta}\right)\right|$, where $J\left(t_{\alpha \beta}\right)$ means the Jacobian of matrix of the partial derivatives of $t_{\alpha \beta}$. Under the above conditions we can write (with the usual abuse of notation) for a given $\stackrel{\triangle}{A} \in \sec \left(L(M) \otimes \bigwedge T^{*} M\right)$,

$$
\stackrel{\triangle}{A}=e_{(\alpha)} \otimes A_{(\alpha)}=e_{(\beta)} \otimes A_{(\beta)}
$$

This formula leaves it clear once again that to start any game with impair forms we must, once we choose a given chart $\left(U_{\alpha}, \varphi_{\alpha}\right)$, to give by convention an orientation $e_{(\alpha)}$ for it and next we must choose a pair form $A_{(\alpha)}=A$ or its negative, i.e., $A_{(\alpha)}=-A$ to build $\stackrel{\triangle}{A}$. This choice depends of course on the applications we have in mind.

\subsection{The Impair Volume Element}

The impair 4-form $\triangle_{g} \in \sec \bigwedge_{-}^{4} T^{*} M$ whose representative in an arbitrary basis $\left\{d x^{\mu}\right\}$ supposed positive is given by

$$
\begin{aligned}
\stackrel{\triangle}{\tau}_{g} & =\frac{1}{4 !} \dot{\boldsymbol{\tau}}_{i_{o} i_{1} i_{2} i_{3}} d x^{i_{1}} \wedge \cdots \wedge d x^{i_{p}}=\stackrel{\mathbf{\tau}}{\tau}_{0123} d x^{0} \wedge \cdots \wedge d x^{3} \\
& =\sqrt{\left|\operatorname{det}\left(g_{i j}\right)\right|} d x^{0} \wedge \cdots \wedge d x^{3},
\end{aligned}
$$

is sometimes called (see, e.g., 20] ) the pseudo volume element27. Now, the representative of this impair form in the basis $\left\{d x^{\prime \mu}\right\}$ is according to the definition

\footnotetext{
${ }^{27}$ Here it becomes obvious what we said in Remark 12 We want of course, that the volume of a compact region $U \subset M$ be a positive number. This implies that we must choose as elements in the trivialization of $L(M) \otimes \bigwedge T^{*} M, 1 \otimes \sqrt{\left|\operatorname{det}\left(g_{i j}\right)\right|} d x^{0} \wedge \cdots \wedge d x^{3}$ or $(-1) \otimes$ $\left(-\sqrt{\left|\operatorname{det}\left(g_{i j}\right)\right|} d x^{0} \wedge \cdots \wedge d x^{3}\right)$ which is simply written as $\stackrel{\triangle}{g}_{g}=\sqrt{\left|\operatorname{det}\left(g_{i j}\right)\right|} d x^{0} \wedge \cdots \wedge d x^{3}$.
} 
just given

$$
\begin{aligned}
& {\stackrel{\triangle^{\prime}}{\mathrm{g}}}_{\mathrm{g}}^{\prime}=\frac{1}{4 !}{\stackrel{\mathbf{\tau}^{\prime}}{i_{o} i_{1} i_{2} i_{3}}}^{\prime} d x^{\prime i_{1}} \wedge \cdots \wedge d x^{\prime i_{p}}={\stackrel{\mathbf{\iota}^{\prime}}{\tau_{0123}}}^{\prime} d x^{\prime 0} \wedge \cdots \wedge d x^{\prime 3} \\
& =\sqrt{\left|\operatorname{det}\left(g_{i j}^{\prime}\right)\right|} d x^{\prime 0} \wedge \cdots \wedge d x^{\prime 3},
\end{aligned}
$$

where we used that $\sqrt{\left|\operatorname{det}\left(g_{i j}^{\prime}\right)\right|}=|\operatorname{det} \Lambda| \sqrt{\left|\operatorname{det}\left(g_{i j}\right)\right|}$ and Eq.(31), i.e.,

$$
{\hat{\tau^{\prime}}}_{0123}=|\operatorname{det} \Lambda| \mathbf{\tau}_{0123} .
$$

Note that Eq.(36) is different from Eq.(15) which defines the transformation rule for the components of a pair volume element.

We recall also that given a chart $(U, \varphi)$ of the atlas of $M$, the integral of an impair $n$-form $\stackrel{\Delta}{\tau}_{g}$ on a compact region $R \subset U \subset M$ is according to de Rham's definition (with $\mathbf{R}=\varphi(R)$ ) given by

$$
\int_{R}{\stackrel{\Delta}{\tau_{g}}}_{\mathbf{R}}:=\int_{\mathbf{R}}{\stackrel{\Delta}{\tau_{0123}}} d x^{0} d x^{1} d x^{2} d x^{3},
$$

and as a result of $E q$. (31) we have in the chart $\left(U^{\prime}, \varphi^{\prime}\right), R \subset U^{\prime}$ and with $\mathbf{R}^{\prime}=\varphi^{\prime}(R)$,

$$
\begin{aligned}
& \int_{R}{\stackrel{\tau_{g}^{\prime}}{\prime}}_{{ }^{\prime}}=\int_{\mathbf{R}^{\prime}}|\operatorname{det} \Lambda| \boldsymbol{\Delta}_{0123} d x^{\prime 0} d x^{11} d x^{\prime 2} d x^{\prime 3} \\
& =\int_{\mathbf{R}} \widehat{\Delta}_{0123} d x^{0} d x^{1} d x^{2} d x^{3},
\end{aligned}
$$

which corresponds to the classical formula for variables change in a multiple integration. Thus the integral of an impair $n$-form on a $n$-dimensional manifold is independent of the orientation of $R$. This is not the case if we try to integrate a pair $n$-form. We briefly recall de Rham's theory [47] of how to integrate pair and impair $p$-forms living on an $n$-dimensional manifold $M$.

Remark 13 Suppose we assign the natural orientation to a 'rectangle' $U^{p} \subset \mathbb{R}^{p}$ by $\tau=d \mathbf{x}^{1} \wedge \cdots \wedge d \mathbf{x}^{p}$ (where $\left\{\mathbf{x}^{i}\right\}$ are Cartesian coordinates for $\mathbb{R}^{p}$ ). It is now a classical result due to de Rham that it is always possible to integrate a pair p-form $\alpha \in \sec \bigwedge^{p} T^{*} M$ over an inner oriented p-chain, i.e., a parametrized submanifold $\psi: U^{p} \rightarrow M$ endowed with an inner orientation [6, 9, 47, 20] $\bar{o}\left(U^{p}\right)$. Indeed, if $\left\{u^{i}\right\}$ are arbitrary coordinates covering $\psi\left(U^{p}\right)$, we have by definition

$$
\begin{aligned}
\int_{\left(\psi\left(U^{r}\right), \bar{o}\left(U^{r}\right)\right)} \alpha & =\int_{\left(U^{r}, \bar{o}\left(U^{r}\right)\right)} \psi^{*} \alpha \\
& :=\bar{o}\left(U^{r}\right) \int_{\left(U^{r}, \bar{o}\left(U^{r}\right)\right)} \psi^{*} \alpha\left(\frac{\partial}{\partial u^{1}}, \ldots, \frac{\partial}{\partial u^{r}}\right) d u^{1} \ldots d u^{r},
\end{aligned}
$$


where $\bar{o}\left(U^{r}\right)=\operatorname{sign} \operatorname{det}\left(\frac{\partial u^{i}}{\partial \mathbf{x}^{j}}\right)$. Of course, if we assign a different orientation $\bar{o}^{\prime}\left(U^{r}\right)=-\bar{o}\left(U^{r}\right)$ to $U^{p}$ we have

$$
\int_{\left(\psi\left(U^{r}\right),-\bar{o}^{\prime}\left(U^{r}\right)\right)} \alpha=-\int_{\left(\psi\left(U^{r}\right), \bar{o}\left(U^{r}\right)\right)} \alpha .
$$

It is now opportune to bethink that any impair $n$-form is always integrable over any compact $n$-dimensional manifold $M$, be it orientable or not. However, it is not always possible to integrate an impair $r$-form $\stackrel{\Delta}{\alpha}$ on a $n$-dimensional manifold over a parametrized submanifold $\psi: U^{r} \rightarrow M$ unless $\psi$ is an outer orientable map, i.e., if we can associate an orientation to $M$ on $\psi\left(U^{r}\right)$. In general it may be not be possible to do that, and thus, we cannot integrate $\stackrel{\Delta}{\alpha}$ over an orientable p-dimensional submanifold $S \subset \psi\left(U^{r}\right)$, unless $S$ is endowed with an outer or transverse orientation, i.e., if at any point of $x \in V, T_{x} M=T_{x} S \oplus N$ (with any $n \in N$ being transverse to $S$, i.e., $n \notin T_{x} S$ ) then each transversal $N$ can be oriented continuously as a function of $x \in V$. Let $\left(x^{1}, \ldots, x^{n}\right)$ be coordinates covering $U \subset M$ such that $S \cap U$ is defined by $x^{\iota}=f\left(x^{1}, \ldots, x^{r}\right)$, $\iota=r+1, \ldots, n$. Of, course the vector fields $\frac{\partial}{\partial x^{\iota}}, \iota=r+1, \ldots, n$, defined in $U$, are transverse to $S$. Given an orientation for $S \cap U$, there always exists a set of vector fields $\left\{\mathbf{e}_{1}, \ldots, \mathbf{e}_{r}\right\}$ in $T(S \cap U)$ that are positively oriented there, which can be extended to all TU by trivially keeping their components constant when moving out of $V$. In this way an outer orientation can be defined in $U$ by saying that $\left\{\mathbf{e}_{1}, \ldots, \mathbf{e}_{r}, \frac{\partial}{\partial x^{r+1}}, \ldots, \frac{\partial}{\partial x^{n}}\right\}$ defines, let us say, the positive orientation on

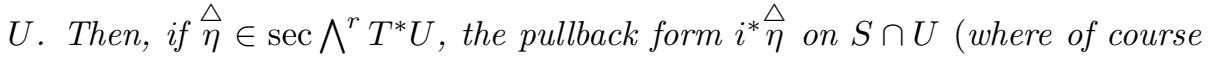
$i: S \rightarrow M$ is the embedding of the submanifold $S$ on $M)$ is well defined, and can be integrated [20]. Finally, recall that the orientations defined by $o(\omega)$ and $\bar{o}\left(U^{r}\right)$ are obviously related, and we do not need further explanation.

Remark 14 It is essential to recall that $\bigwedge_{-} T^{*} M$ is not closed under the operation of exterior multiplication and indeed to have a closed algebra (with that product) we need to take into account that the exterior multiplication of forms of the same parity is always a pair form and the exterior multiplication of forms of different parities is always an impair form. Also, the scalar product of forms of the same parity gives a pair 0-form and the scalar product of forms of different parities gives an impair 0-form. Moreover, the differential operator d preserves the parity of forms.

Remark 15 If we insist in using pair and impair forms for formulating the differential equations of motion of a given theory, e.g., in a formulation of electromagnetism in an orientable spacetime (something that at this point the reader must be convinced that it is not necessary at all, as it clear from the presentation given above) we need to introduce an impair Hodge star operator. 


\subsection{The Impair Hodge Star Operator}

Definition 16 Let $\stackrel{\triangle}{\tau}_{g} \in \sec \bigwedge_{-}^{4} T^{*} M$ be an impair volume form. The impair Hodge star operator is the map

$$
\begin{aligned}
& \underset{\stackrel{\tau}{\tau}_{g}}{\star}: \bigwedge^{p} T^{*} M \rightarrow \bigwedge_{-}^{4-p} T^{*} M, \\
& \underset{\hat{\tau}_{g}}{\star}: \bigwedge_{-}^{p} T^{*} M \rightarrow \bigwedge^{4-p} T^{*} M
\end{aligned}
$$

such that for any $A_{p} \in \sec \bigwedge^{p} T^{*} M$ and $\stackrel{\triangle}{B}_{p} \in \sec \bigwedge_{-}^{p} T^{*} M$, we have

$$
\begin{aligned}
& \underset{\stackrel{\sim}{\tau}_{g}}{\star} A_{p}:=\tilde{A}_{p} \stackrel{\Delta}{\tau}_{g}^{\omega},
\end{aligned}
$$

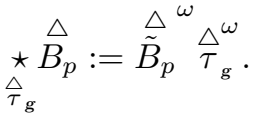

Note that in Eq.(43) the Clifford product for the representatives of the impair forms is well defined (since according to Definition 9 each representative is an even form). Given the existence of impair and pair forms, many authors, e.g., [20, 43, 44, 45, 35] advocate that even in an orientable manifold the formulation of electromagnetism must necessarily use besides the pair field strength $F \in$ $\sec \bigwedge^{2} T^{*} M$ an impair exact 3 -form $\overrightarrow{\mathbf{J}} \in \sec \bigwedge_{-}^{3} T^{*} M$, which then defines the excitation field as an impair 2-form $\stackrel{\Delta}{G} \in \sec \bigwedge_{-}^{3} T^{*} M$. We have thus for the vacuum situation

$$
\begin{aligned}
& d F=0, d G=-\stackrel{\Delta}{\mathbf{J}}, \\
& \stackrel{\Delta}{G}=\underset{\substack{\tau_{g} \\
\tau_{g}}}{\Delta} .
\end{aligned}
$$

Remark 17 Authors [20, 43, 44, 45, 35, (since the classical presentation of 57]) offers as the main argument for the necessity of using an impair $\Delta \overrightarrow{\mathbf{J}} \in$ sec $\bigwedge^{3} T^{*} M$ in electromagnetism the following statement (which takes into account the Remark 131): "the value of the charge

$$
Q=\int_{S} \stackrel{\triangle}{\mathbf{J}}
$$

contained in a compact spacelike hypersurface $S \subset M$ must be independent of 
the orientation of $S \sqrt{28}$ and indeed, taking into account that $\stackrel{\triangle}{\mathbf{J}}=\underset{\substack{\tau_{g} \\ \star}}{\text {, where } J}$ is given by Eq.(2) we must have

$$
\int_{S} \stackrel{\triangle}{\mathbf{J}}=\sum_{i} q^{(i)}
$$

However, it is our view that the above argument is not a solid one. First, there is no empirical evidence that any spacelike surface $S \subset M$ where a real current is integrated does not possess an inner orientation that may be make consistent with the orientation of $M$. Thus, empirical evidence asserts that we may restrict ourself to only positively oriented charts (see also Section 4.3). But, even if we do not want to restrict ourselves to the use of positively oriented charts we must not forget that to perform the integral $\int_{S} \overrightarrow{\mathbf{J}}$ using a chart $(U, \varphi)$, $S \subset U$ with coordinates $\left\{x^{\mu}\right\}$ we must choose an orientation (recall Remark

13) for that chart and pick a specific choice for the pair form representing $\stackrel{\triangle}{\mathbf{J}}$. Suppose we make the convention that the orientation of $d x^{0} \wedge d x^{1} \wedge d x^{2} \wedge d x^{3}$ is positive. At our disposal there are $\mathbf{J}$ and $-\mathbf{J}$. Which one to choose? The answer is obvious, we choose $\mathbf{J}$, for in this case we will have $\int_{S} \stackrel{\Delta}{\mathbf{J}}=\sum_{i} q^{(i)}$. This means that the attribution of the charge parameters to particles need in order to define the current depends on a convention, the one described above.

What happens if we represent the current by a pair form $\mathbf{J} \in \sec \bigwedge^{3} T^{*} M$ ? In this case the integral

$$
\int_{S} \mathbf{J}
$$

does depend on the orientation of the chart used for its calculation. Suppose that as in the case of the integration of the impair form we use a chart $(U, \varphi), S \subset U$ with coordinates $\left\{x^{\mu}\right\}$. Which orientation should we give to $d x^{0} \wedge d x^{1} \wedge d x^{2} \wedge d x^{3}$ ? The answer is obvious. We must choose an orientation (the positive one) such that

$$
\int_{S} \mathbf{J}=\sum_{i} q^{(i)}
$$

So, in both cases (use of impair or pair current forms) to start the evaluation process we need to make a convention in order to fix the charge parameters of

\footnotetext{
${ }^{28}$ Once we use impair forms, following [20] we may say that charge is a scalar. However, take care, in, e.g., [35] charge is said to be a pseudo-scalar. This apparent confusion comes out because in [35] it is discussed the properties of charge and of other electromagnetic quantities under a active parity operation and time reversal operators interpreted as appropriated mappings $\mathbf{p}: M \rightarrow M$ and $\mathbf{t}: M \rightarrow M$, where $M \simeq \mathbb{R}^{4}$ is the manifold entering in the structure of Minkowski spacetime. In that case we can show, e.g., that if $\overrightarrow{\mathbf{J}}$ is an impair 2-form than

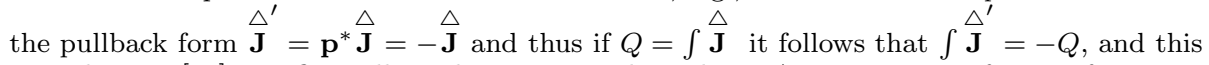
according to [35] justifies calling charge a pseudo-scalar. As we see a confusion of tongues are also present in our subject. We are going to discuss more details of this particular issue in another publication.
} 
the charges that enter the definition of current such that Eq.(46) and Eq.(48) are true.

Now, what happens if someone using pair forms decides to calculate $\int_{S} \mathbf{J}$ using a chart with a different orientation than the one previously used to fix the charge parameters of the particles? The answer is that he will find that the value of the integral given by Eq.(47) will be now $-\sum_{i} q^{(i)}$ ? Is this a puzzle? Of course not, the value is negative because he is using a different convention than the previous one.

What we should ask is that if this break of convention changes the physics of electromagnetic phenomena? No, what happens is only that what was called a positive charge will now be called a negative charge and what was called a negative charge will now be called a positive one. No empirical fact will change, only some names. This is so because this change of names does not change any prediction of the theory concerning the motion of charged particles that besides the coupling parameter $q$ also carries a coupling parameter $m$. We shall explicitly demonstrate this statement in Section 6 after we introduce the Clifford bundle formulation of electromagnetism. Here we emphasize again: the sign of a charge can be given meaning only by making it to interact with a charge that has been defined by convention as being positive (or negative) 29 .

\subsection{Teaching Aliens What is Right Hand and What is Left Hand}

The previous considerations of Remark [17 are valid only if the universe we live does not have regions composed of what we here called antimatter. Indeed, let us recall one of Feynman's stories (at page 103 of [18]) on the subject. Suppose we are in contact with some alien species, but only by the exchange, say of radio signals. Any intelligible communication needs a language and we suppose to build one doing something similar to the one proposed in the SETI program, starting with telling aliens what we mean by prime numbers and progressing to pictures, physics, and chemistry information. The concept of distance may be grasped by the aliens, e.g., by telling then how tall we are (in the mean), by expressing such number in mutually understood wavelengths of light. They can use that information to tell us how tall they are. We can also teach the aliens the concept of a man lifetime by expressing such number by the number of ticks of a light-frequency clock. To make agreement of some physical conventions and also to explain some social procedures among men (e.g., the fact that we shake hands when we meet, by extending our right hand) we need to explain them what is a right hand. How to do that?

As well known, until 1957 we could not answer that question. But, after the discover of the experiments showing parity violation in that year, we can explain to the aliens what the right hand is by asking them to repeat the original experiment done by $\mathrm{Wu} 67$, et al with ${ }^{60} \mathrm{Co}$, but in such a way that they must

\footnotetext{
${ }^{29}$ This point is well discussed in [62 63] where the author uses an interesting homological approach in the formulation of the electromagnetic laws.
} 
turn their apparatus (including the magnetic field generator in use) until the electrons come out in the downward direction, which we may define as the one of their local gravity pull. In such a situation the rotating nucleus will be with their spins up, i.e., rotating in the anti-clockwise direction as seen from the top. Before someone says that the aliens cannot see the ${ }^{60}$ Co rotating we describe how we can teach them to amplify this anti-clockwise rotation (as seen from the top) in order that it becomes macroscopically visible. Indeed, all they need is to follow the following instructions. (A) Take a spherical conductor of radius $a$ in electrostatic equilibrium with an uniform superficial charge density with total charge $Q$ (i.e., charge as the ones carried by the atomic nucleus of the ${ }^{60} \mathrm{Co}$ ) and which is magnetized with its dipole magnetic moment of modulus $\varsigma$ oriented in the same direction (the $\hat{\mathbf{z}}$-direction) of the magnetic moment of the ${ }^{60} \mathrm{Co}$ nucleus in his repetition of Wu's experiment. Such charged magnet has electromagnetic angular momentum stored in its electromagnetic field given by $\mathbf{L}_{e m}=\frac{2}{9} \varsigma Q a^{2} z^{30}$. (B) The aliens are next instructed to discharge the magnet (suspended from the roof with an insulator) through the south pole. This makes the magnet to rotate anti-clockwise as seen from the top, in order to conserve the total angular momentum of field plus matter. Indeed a simple calculation [60, 24] shows that the mechanical angular momentum acquired by the sphere once completely discharged is $31 \mathbf{L}_{m e c}=\frac{2}{9} \varsigma Q a^{2} \hat{\mathbf{z}}$.

Of course, Feynman cautions us (page 107 of [18) that after lots of communication if we finally can go into space and meet the aliens counterpart, if it happens that their leader extends its left hand to shake, stop immediately because that is proof that he is made of antimatter. This, of course, is because Wu's parity violation experiment constructed of antimatter would give the opposite result.

Feynman's story is important four the objectives of this paper because it shows that the charge argument is indeed a very week one. To fix the signal of the charge parameters that label particles and to describe their currents, we need to start with a convention, we need a local orientation, we need to know what a right hand is 32

\subsection{Electromagnetism in a medium}

Classical electromagnetism in a general medium is a very complicated subject since admitting with Feynman that the only the fundamental physical fields $F$ and the current $J$ generated by the particles carriers must enter the game are

\footnotetext{
${ }^{30}$ This stored angular momentum in static electric plus magnetic field has been experimentally verified in 23 .

${ }^{31}$ This result is obtained once we neglect the magnetic field associated with the discharging current and displacement current associated with the collapsing electric field, something justifiable if the current is small. If the current is not small some angular momentum will be carried by the radiation field, but of course at the end of the discharging process the sphere will be rotating.

${ }^{32}$ More recently Elitzur and Shinitzky [14] showed how to teach aliens what is right and what is left using the space asymmetry of molecules ( $\mathrm{L}$ and $\mathrm{D}$ amino acids). However, to their method Feynman's caution also applies.
} 
we immediately involved in an almost intractable many body system. However, it seems empirical fact that the equations

$$
\begin{aligned}
d F & =0, d G=-\mathbf{J}, \\
G & =\chi(F)
\end{aligned}
$$

or

$$
\begin{aligned}
d F & =0, d \overleftrightarrow{G}=-\overrightarrow{\mathbf{J}}, \\
\stackrel{\leftrightarrow}{G} & =\grave{\chi}(F)
\end{aligned}
$$

describes essentially all macroscopic electromagnetic phenomena on any medium contained in a world tube in $U \subset M$. In those equations $\kappa$ and $\Delta$ are multiform functions [15, 16] of the multiform variable $F$, i.e., for each $x \in U \subset M$ we have

$$
\begin{aligned}
& \left.\chi\right|_{x}: \bigwedge^{2} T_{x}^{*} M \rightarrow \bigwedge^{2} T_{x}^{*} M, \\
& \left.\grave{\chi}\right|_{x}: \bigwedge^{2} T_{x}^{*} M \rightarrow \bigwedge_{-}^{2} T_{x}^{*} M .
\end{aligned}
$$

Consider, e.g., Eq.(51b). The multiform function $\hat{\chi}$ is phenomenologically described, e.g., using coordinates in the ELPG by 31, 33.

$$
\stackrel{\triangle}{G}^{\mu \nu}=\frac{1}{2}\left(\stackrel{\triangle}{\chi}^{\mu \nu \rho \sigma} F_{\rho \sigma}+\stackrel{\triangle}{\varsigma}^{\mu \nu \rho \sigma \iota \zeta} F_{\rho \sigma} F_{\iota \zeta}+\cdots\right) .
$$

A medium for which $\stackrel{\triangle}{\varsigma}^{\mu \nu \rho \sigma \iota \zeta} \neq 0$ is called nonlinear. For what follows we restrict our considerations only to linear media. In that case the constitutive multiform function $\grave{\chi}$ is a $(2,2)$-extensor field [15, 16] and we have the decomposition [28]

$$
\stackrel{\triangle}{\chi}^{\mu \nu \rho \sigma}={ }^{(1)} \grave{\chi}^{\mu \nu \rho \sigma}+{ }^{(2)} \grave{\chi}^{\mu \nu \rho \sigma}+\stackrel{\triangle}{a}^{(3)} \varepsilon^{\mu \nu \rho \sigma},
$$

where ${ }^{(1)} \chi^{\mu \nu \rho \sigma}$ is a trace free symmetric part (with 20 independent components), ${ }^{(2)} \triangle^{\mu \nu \rho \sigma}$ is the antisymmetric part (with 15 independent components) and $\varepsilon^{\mu \nu \rho \sigma}$

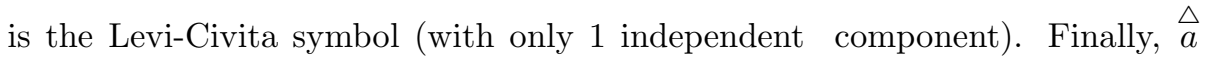
is an impair 0-form field (also called a pseudo-scalar function) called the axion field. It has been a conjecture (called Post conjecture [43]) that $\stackrel{\Delta}{a}$ must be null for any medium.

However, recently it has been found that for $\mathrm{Cr}_{2} \mathrm{O}_{3}, \stackrel{\Delta}{a} \neq 0$. In [27] it is claimed that this fact even proves that we must use impair forms in the description of electromagnetism. However, those authors forget the following observation that can be found at page 22 of de Rham's book: 
"Si la variété $V$ est orienté, c' est-à-dire si elle est orientable et si l" on a choisi une orientation $\varepsilon$, à toute forme impaire $\alpha$ est associée une forme paire $\varepsilon \alpha$. Par la suite, dans le cas d'une varieté orientable, en choissant une foi pour toutes une orientation, il serai possible d'éviter l'emploi des formes impaires. Mais pour les variétés non orientables, ce concept est réellement utile et naturel."

Now, for de Rham, an orientation $\varepsilon$ is an impair 0-form field (i.e., an axion field) defined in the manifold $M$ (that in his book is called $V$ ) ${ }^{33}$. So, all that the authors of [27] need to do in order to have only pair forms in their formulation of the electromagnetism of $\mathrm{Cr}_{2} \mathrm{O}_{3}$ is to multiply their impair objects by $\stackrel{\Delta}{a}$. So, contrary to popular believe the existence of an axion field does not imply that spacetime is non oriented. Quite the contrary is what is true.

For some media where ${ }^{(2)} \grave{\chi}^{\mu \nu \rho \sigma}=0$, and $\stackrel{\Delta}{a}=0$ we even find that the constitutive extensor may be described by 43.

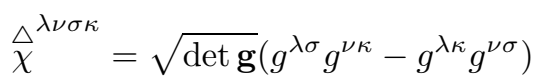

where $g^{\lambda \sigma} g_{\lambda \nu}=\delta_{\nu}^{\sigma}$ and $g_{\mu \nu}$ are the components of an effective metric field $\mathrm{g}=g_{\mu \nu} d \mathrm{x}^{\mu} \otimes d \mathrm{x}^{\nu}$ for $M$. A particular medium with such characteristic is the vacuum in the presence of a gravitational field, but here we do not want to go deeply on this issue.

Remark 18 Until to this point the complete Minkowski spacetime structure $\left(M, \mathbf{g}, D, \tau_{\mathrm{g}}, \uparrow\right)$ did not enter our formulation of electromagnetism. So, let us remark, first of all that from the point of view of an experimental physicist the parallelism rule defined by $D$ is essential, since it is this parallel transport rule that permits him(her), e.g., to make to parallel filamentary currents and find their interaction behavior (as long ago did Ampere).

From a mathematical point of view, the connection $D$ enter in our formulation of electromagnetism through the introduction of the Dirac operator acting on sections of the Clifford bundle $\mathcal{C} \ell(M, g)$.

Remark 19 Also, for media where the constitutive extensor can be put in the form given by Eq.(54) we can give an intrinsic presentation of electromagnetism using the Clifford bundle formalism by introducing an effective Lorentzian spacetime $\left(M, \mathbf{g}, \nabla, \tau_{\mathbf{g}}, \uparrow\right)$ where now, $\nabla$ denotes the non-flat34 Levi-Civita connection of $\mathbf{g}$, an effective Lorentzian metric determined by the constitutive tensor of that effective spacetime.

Remark 20 Before ending this section we have an important observation yet, concerning the metric free formulation of electromagnetism as presented, e.g., in [28. There, it is admitted that $M$ is an oriented connected, non compact,

\footnotetext{
${ }^{33}$ The reader can easily convince himself that this definition is equivalent to the one given in terms of an impair and a pair volume 4 -forms $\stackrel{\Delta}{\tau}_{g}$ and $\tau_{g}$. Indeed, take $\varepsilon=\stackrel{\Delta}{\tau}_{g} \cdot \tau_{g}$.

${ }^{34}$ This term only means that the Riemann tensor $\mathbf{R}(\nabla) \neq 0$.
} 
paracompact Hausdorff space. The authors say that a manifold with those characteristics always permits a codimension-1 foliation, 35, a statement that is true [37. However without any additional structure we cannot see how to foliate spacetime $M$ as time $\times$ space $(\mathbb{R} \times S)$, because we do not know a prior how to choose the dimension that represents time. In [28] the authors quickly introduce a global vector field $\mathbf{n}$ transverse to the folia, and the 3-dimensional manifold $S$ of the foliation is defined by a manifold function $\sigma: M \rightarrow \mathbb{R}$ such that $\sigma(x)=$ constant and $\mathbf{n}\lrcorner d \sigma=0$. It seems clear for us that $\mathbf{n}$ and $\Omega=d \sigma$ are nothing more than the universal vector field and the universal 1-form field defining the structure of absolute space and absolute time in the structure of Newtonian theory when that theory is formulated as a spacetime theory (for details, see [51]). Those observations can be translated in simple words: contrary to some claims only the bare structure of $M$ is not enough for a formulation of electromagnetic theory as a physical theory.

\section{The Clifford Bundle Formulation of Electro- magnetism}

In a medium described by an effective Lorentzian spacetime $\left(M, \mathbf{g}, \nabla, \tau_{\mathbf{g}}, \uparrow\right)$ we may present the equations of electromagnetic theory as a single equation using the Clifford bundle $\mathcal{C} \ell(M, \mathrm{~g})$ of pair differential forms. We recall [54] that in the Clifford bundle formalism the so called Dirac operator 36 acting on sections of $\mathcal{C} \ell(M, \mathrm{~g})$ is given by

$$
\partial=\partial \wedge+\partial\lrcorner
$$

It can be shown (see, e.g., [54]) that for a Levi-Civita connection we have $\boldsymbol{\partial} \wedge=d$ and $\boldsymbol{\partial}\lrcorner=-\delta$, where $\delta$ is the Hodge coderivative operator, such that for any $A_{p} \in \sec \bigwedge^{p} T^{*} M \hookrightarrow \mathcal{C} \ell(M, \mathrm{~g})$ its action is given by:

$$
\delta A_{p}=(-1)^{p}{ }_{\tau_{\mathbf{g}}}^{-1} d \underset{\tau_{\mathbf{g}}}{\star} A_{p}
$$

We also have that:

$$
\begin{aligned}
-\delta A_{p} & \left.=\partial\lrcorner A_{p}=\theta^{\mu}\right\lrcorner\left(\nabla_{e_{\mu}} A_{p}\right), \\
d A_{p} & =\partial \wedge A_{p}=\theta^{\mu} \wedge\left(\nabla_{e_{\mu}} A_{p}\right),
\end{aligned}
$$

and those expressions permit the simplification of many calculations. Recalling that $G=\underset{\tau_{\mathbf{g}}}{\star} F$ we get that $d G=-\mathbf{J}$ can be written defining $J=\underset{\tau_{\mathbf{g}}}{\star} \underset{ }{-1} \mathbf{J} \in$

\footnotetext{
${ }^{35}$ It admits also a 1-dimension foliation.

${ }^{36}$ Please, do not confound the Dirac operator to the spin-Dirac operator which acts on sections of a spinor bundle. See details, e.g., in [54]. Here we recall that in an arbitrary basis $\left\{e_{\mu}\right\}$ for $T U \subset T M$, and $\left\{\theta^{\mu}\right\}$ for $T^{*} U \subset T^{*} M \subset \mathcal{C} \ell(M, \mathrm{~g})$, the operator is given by $\boldsymbol{\partial}:=\theta^{\mu} \nabla_{e_{\mu}}$. Take notice that $\mathbf{g}=g^{\mu \nu} e_{\mu} \otimes e_{\nu}$ if $\mathbf{g}=g_{\mu \nu} d x^{\mu} \otimes d x^{\nu}$, with $g^{\mu \nu} g_{\alpha \nu}=\delta_{\alpha}^{\mu}$.
} 
$\sec \bigwedge^{1} T^{*} M \hookrightarrow \mathcal{C} \ell(M, \mathrm{~g})$ as $\delta F=-J$. Indeed, we have,

$$
\begin{aligned}
d \underset{\tau_{\mathbf{g}}}{\star} F & =-\mathbf{J}, \\
\star_{\tau_{\mathbf{g}}}^{-1} d \underset{\tau_{\mathbf{g}}^{\star}}{\star} F & =-\star_{\tau_{\mathbf{g}}}^{-1} \mathbf{J}, \\
\delta F & =-J
\end{aligned}
$$

Then, the two equations $d F=0$ and $\delta F=-J$ can be summed if we suppose (as it is licit to do in the Clifford bundle $\mathcal{C} \ell(M, \mathrm{~g})$ ) that $F, G \in \sec \bigwedge^{2} T^{*} M \hookrightarrow$ $\mathcal{C} \ell(M, \mathrm{~g})$ and $\mathbf{J} \in \sec \bigwedge^{3} T^{*} M \hookrightarrow \mathcal{C} \ell(M, \mathrm{~g})$ and we get Maxwell equation ${ }^{37}$

$$
\partial F=J .
$$

Remark 21 We now show that Eq.(58) can also be obtained directly from the de Rham formulation of electromagnetism that uses pair and impair forms. Indeed, all that is need is to verify that the formula $d \overleftrightarrow{G}=-\overrightarrow{\mathbf{J}}$ in Eq.(44) - where $\stackrel{\Delta}{G}$ $=\star F-$ can be written as $\delta F=-J$. Indeed, we have

$$
\stackrel{\triangle}{\tau_{\mathrm{g}}}
$$

$$
\begin{aligned}
& d \overleftrightarrow{G}=-\stackrel{\triangle}{\mathbf{J}}, \\
& d \underset{\substack{\tau_{\mathbf{g}}}}{\star} F=-\stackrel{\triangle}{\mathbf{J}} \text {, }
\end{aligned}
$$

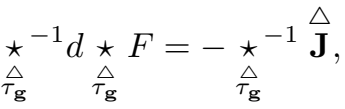

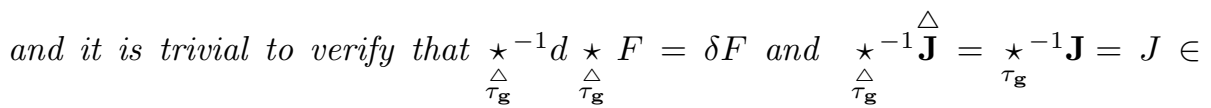
sec $\bigwedge^{1} T^{*} M$. We conclude that the Clifford bundle formulation of electromagnetism given by Maxwell equation (Eq.(44)) is general enough to permit the two formulations of electromagnetism given above.

We are now ready to complete the formulation of electrodynamics as a physical theory. We restrict our presentation here in the case where the existence of the gravitational field must be ignored. As such our formulation will use the Minkowski spacetime structure introduced above and the Clifford bundle $\mathcal{C} \ell(M, g)$.

\footnotetext{
${ }^{37}$ No misprint here! Parodying Thirring 61 that said that the equations $d F=0$ and $\delta F=0$ were the $20^{t h}$ century presentation of Maxwell equations, we say that the single equation $\partial F=J$ is the $21^{\text {th }}$ century presentation of Maxwell equations.
} 


\section{The Energy-momentum 1-forms of the Elec- tromagnetic and the Matter Fields}

We start from Maxwell equation (with $J$ the current of charged particles introduced by Eq.(20)

$$
\partial F=J
$$

where in what follows, $\boldsymbol{\partial}=\theta^{\alpha} D_{e_{\alpha}}=\gamma^{\mu} \frac{\partial}{\partial x^{\mu}}$ is the Dirac operator written with a general pair of dual basis $\left\{e_{\alpha}\right\}$ and $\left\{\theta^{\alpha}\right\}$ for $T U \subset T M$ and $T^{*} U \subset T^{*} M$ and with the basis $\left\{\frac{\partial}{\partial \mathrm{x}^{\mu}}\right\}$ and $\left\{\gamma^{\mu}=d \mathrm{x}^{\mu}\right\}$ for $T M$ and $T^{\star} M$, with $\left\{\mathrm{x}^{\mu}\right\}$ coordinates in the Einstein-Lorentz-Poincaré gauge. Given Eq.(59) its reverse is

$$
\tilde{F} \overleftarrow{\partial}=J
$$

where $\tilde{F} \overleftarrow{\boldsymbol{\partial}}:=\left(D_{e_{\alpha}} \tilde{F}\right) \theta^{\alpha}=\left(\frac{\partial}{\partial \mathrm{x}^{\mu}} \tilde{F}\right) \gamma^{\mu}$. Multiplying Eq.(159) on the left by $\tilde{F}$ and Eq.(60) on the right by $F$ and summing the resulting equations we get

$$
\frac{1}{2}[\tilde{F}(\boldsymbol{\partial} F)+(\tilde{F} \overleftarrow{\boldsymbol{\partial}}) F]=\frac{1}{2}(\tilde{F} J+J F)
$$

Now, let $n=n^{\alpha} \gamma_{\alpha} \in \sec \bigwedge^{1} T^{*} M \hookrightarrow \mathcal{C} \ell(M, g)$ and $\boldsymbol{\partial}_{n}=\gamma^{\alpha} \frac{\partial}{\partial n^{\alpha}}$ acting on multiform functions of the multiform variable $n$. Consider moreover the extensor field $38 T(n)=\frac{1}{2} \tilde{F} n F$. Now, observe that if we apply $\gamma^{\alpha} \cdot \boldsymbol{\partial}_{n}$ to the multiform function $\mathbf{f}(n)=\frac{\partial n}{\partial \mathbf{x}^{\alpha}}$ we get 39

$$
\begin{aligned}
\gamma^{\alpha} \cdot \boldsymbol{\partial}_{n} \frac{\partial n}{\partial \mathrm{x}^{\alpha}} & =\eta^{\alpha \mu} \frac{\partial}{\partial n^{\mu}}\left(\frac{\partial}{\partial \mathrm{x}^{\alpha}} n^{\beta} \gamma_{\beta}\right) \\
& =\eta^{\alpha \mu} \frac{\partial}{\partial \mathrm{x}^{\alpha}}\left(\delta_{\mu}^{\beta} \gamma_{\beta}\right)=0 .
\end{aligned}
$$

Using Eq.(62) we can write the first member of Eq.(61) as

$$
\begin{aligned}
& \tilde{F} \gamma^{\lambda} \frac{\partial F}{\partial \mathrm{x}^{\lambda}}+\frac{\partial \tilde{F}}{\partial \mathrm{x}^{\lambda}} \gamma^{\lambda} F \\
& =\gamma^{\lambda} \cdot \boldsymbol{\partial}_{n}\left(\tilde{F} n \frac{\partial F}{\partial \mathrm{x}^{\lambda}}+\tilde{F} \frac{\partial n}{\partial \mathrm{x}^{\lambda}} F+\frac{\partial \tilde{F}}{\partial \mathrm{x}^{\lambda}} n F\right) \\
& =\gamma^{\lambda} \cdot \boldsymbol{\partial}_{n} \frac{\partial}{\partial \mathrm{x}^{\lambda}}(\tilde{F} n F) \\
& =\frac{\partial}{\partial \mathrm{x}^{\lambda}}\left(\tilde{F} \gamma^{\lambda} F\right)
\end{aligned}
$$

\footnotetext{
${ }^{38}$ An extensor field $T: \bigwedge^{1} T^{*} M \rightarrow \bigwedge^{1} T^{*} M, n \mapsto T(n)$ is a linear multiform function of the form field $n$.

${ }^{39}$ See details on the derivation of multiform functions in [40].
} 
On the other hand the second member of Eq.(61) is just $-J\lrcorner F$. So, we have

$$
\left.\frac{\partial}{\partial \mathrm{x}^{\alpha}} T^{\alpha}=J\right\lrcorner F,
$$

with the $T^{\alpha} \in \sec \bigwedge^{1} T^{*} M \hookrightarrow \mathcal{C} \ell(M, g)$ given by 40

$$
T^{\alpha}=\frac{1}{2} F \gamma^{\alpha} \tilde{F}
$$

being the pair energy-momentum 1-forms of the electromagnetic field. Indeed, a simple calculation shows that

$$
T^{\alpha \beta}=T^{\alpha} \cdot \gamma^{\beta}=\eta^{\alpha \mu} F_{\mu \lambda} F^{\lambda \beta}+\frac{1}{4} \eta^{\alpha \beta} F_{\mu \nu} F^{\mu \nu},
$$

a well known formula. Now, we contract Eq.(64) on the left with $\gamma^{\alpha}$ getting

$$
\left.\left.\partial\lrcorner T^{\alpha}=\gamma^{\alpha}\right\lrcorner(J\lrcorner F\right)
$$

Now 41

$$
\begin{aligned}
\left.\left.\gamma^{\alpha}\right\lrcorner(J\lrcorner F\right) & \left.=\left(\gamma^{\alpha} \wedge J\right)\right\lrcorner F=-\left(\gamma^{\alpha} \wedge J\right) \cdot F \\
& \left.=-F \cdot\left(\gamma^{\alpha} \wedge J\right)=-\left(\gamma^{\alpha}\right\lrcorner F\right) \cdot J,
\end{aligned}
$$

and Eq. (67) becomes (taking into account that $\partial\lrcorner T^{\alpha}=-\delta T^{\alpha}$ )

$$
\left.\delta T^{\alpha}=\left(\gamma^{\alpha}\right\lrcorner F\right) \cdot J
$$

Defining $f^{\alpha} \in \sec \bigwedge^{4} T^{*} M$ as the pair density of force by

$$
\left.f^{\alpha}=\left[\left(\gamma^{\alpha}\right\lrcorner F\right) \cdot J\right] \tau_{g},
$$

where $\tau_{g}$ is a pair metric volume element, we obtain (the equivalent expressions)

$$
\begin{aligned}
f^{\alpha} & \left.\left.\left.\left.\left.=\left[\left(\gamma^{\alpha}\right\lrcorner F\right) \cdot J\right)\right] \tau_{g}=\underset{\tau_{\mathrm{g}}}{\star}\left[\left(\gamma^{\alpha}\right\lrcorner F\right)\right\lrcorner J\right]=\left(\gamma^{\alpha}\right\lrcorner F\right) \wedge \underset{\tau_{\mathrm{g}}}{\star} J \\
& \left.=\left(\gamma^{\alpha}\right\lrcorner F\right) \wedge \mathbf{J},
\end{aligned}
$$

where, in particular, the last one is the pair density of force.

Remark 22 Note that we could return to Eq. (22) and get an impair density of force simply by replacing the pair volume element $\tau_{g}$ by an impair volume element $\stackrel{\Delta}{\tau}_{g}$, i.e., defining

$$
\left.\stackrel{\triangle}{f}^{\alpha}:=\left[\left(\gamma^{\alpha}\right\lrcorner F\right) \cdot J\right] \stackrel{\Delta}{\tau_{g} .}
$$

\footnotetext{
${ }^{40}$ An equation equivalent to Eq. 65] has been discovered by M. Riesz [48].

${ }^{41}$ The sequence of identities in Eq. (68) may be found in Section 2.4.2 of [54].
} 
Such a formula was postulated in the presentation of electromagnetism in [28] However, as we just saw, that postulate for the coupling of the field $F$ with the current $\mathbf{J}$ is not necessary in our approach, since that force density is already contained in Maxwell equation (Eq.(59)). We now write Eq.(69) as

$$
\left.d \underset{\tau_{g}}{\star} T^{\alpha}=\left(\gamma^{\alpha}\right\lrcorner F\right) \wedge \underset{\tau_{g}}{\star} J
$$

or

$$
\left.d \underset{\widehat{\tau}_{g}}{\star} T^{\alpha}=\left(\gamma^{\alpha}\right\lrcorner F\right) \wedge \underset{\widehat{\tau}_{g}}{\star} J
$$

and both, of course, in components reads

$$
\partial_{\nu} T^{\alpha \nu}=J_{\nu} F^{\nu \alpha}
$$

\subsection{Total Energy-Momentum Conservation and the Knock- down of the Charge Argument}

Eq. (175) asserts that the energy momentum tensor of the electromagnetic field is not conserved. We expect that the total energy-momentum of the field and the charged particles is conserved since there is not a single experiment in Physics contradicting it, and so without much ado, recalling the definition of the pair energy-momentum 1-forms of the charged matter, the $\mathbf{T}^{\alpha}$ given by Eq.(7) we postulated that:

$$
\delta T^{\alpha}+\delta \mathbf{T}^{\alpha}=0
$$

which may be written as:

$$
\left.\left(\gamma^{\alpha}\right\lrcorner F\right) \wedge \underset{\tau_{g}}{\star} J=-d \underset{\tau_{g}}{\star} \mathbf{T}^{\alpha} .
$$

or

$$
\left.\left(\gamma^{\alpha}\right\lrcorner F\right) \wedge \underset{\substack{\tau_{g}}}{\star} J=-d \underset{\stackrel{\Delta}{\tau}_{g}}{\star} \mathbf{T}^{\alpha} .
$$

Eq.(777) is of course the Lorentz force law, and all objects in it are pair forms. On the other hand Eq.(78) is also an expression of the Lorentz force law and there are objects on it that are pair and others that are impair forms. Both equations give in our opinion the correct description of physical phenomena. However let us analyze here Eq.(77), since it permits us to knockdown again the charge argument [20, 43, 44, 45, 35] which says that the density of current

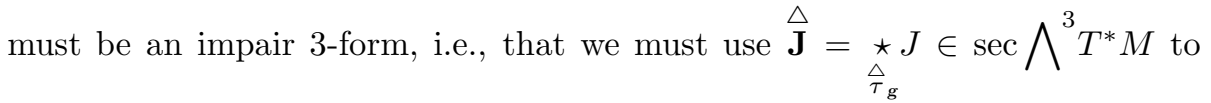
calculate without ambiguity the charge in a certain region, say $U \in M$. To see this, recall that the total energy-momentum 1-form of matter in $U$ at time $\mathrm{x}^{0}=t$ in an inertial reference frame $\mathbf{I}=\frac{\partial}{\partial \mathrm{x}^{0}}$ is

$$
P(t)=\left(\int_{U} \underset{\tau_{g}}{\star} \mathbf{T}^{\alpha}\right) \gamma_{\alpha}
$$


Now, if we change the orientation of $U$ two things happen. What was called electric charge $q^{(i)}=\left.\int_{U}^{\underset{\tau_{g}}{\star} J^{(i)}}\right|_{\sigma^{(i)}}$ of the $i$-particle changes into $-q^{(i)} . F$ changes into $-F$ (despite the fact that it is a pair form) because of the formula used to calculate it (see Appendix). If we are interested in the motion of only a single small particle modeled by a thin world tube in an external field $F$, when integrating Eq.(79) we get that what we originally called energy at time $t$, $E^{(i)}(t)=\left.\int_{U} \underset{\tau_{\mathrm{g}}}{\star} \mathbf{T}^{0}\right|_{\sigma^{(i)}}$ of $i$-particle changes into $-E^{(i)}(t)$. This sign changes in Eq. (179) is compensated by the sign change that occurs in $\left.\int_{U}\left(\gamma^{\alpha}\right\lrcorner F\right) \wedge \underset{\tau_{g}}{\wedge} J_{(i)}$ and it follows that the prediction for the trajectory of that particle does not change if we change the orientation of $U$. And since trajectories of particles are all what are experimentally detected, it follows that the formulation of electrodynamics with only pair forms is compatible with the experimental facts.

\section{The Engineering Formulation of Electromag- netism Without Axial Vector Fields}

We recall (see details in [54]) that for any $x \in M, \mathcal{C} \ell\left(T_{x}^{*} M, g_{x}\right) \simeq \mathbb{R}_{1,3} \simeq \mathbb{H}(2)$, is the so called spacetime algebra. The even elements of $\mathbb{R}_{1,3}$ close a subalgebra called the Pauli algebra. That even subalgebra is denoted by $\mathbb{R}_{1,3}^{0} \simeq \mathbb{R}_{3,0} \simeq$ $\mathbb{C}(2)$. Also, $\mathbb{H}(2)$ is the algebra of the $2 \times 2$ quaternionic matrices and $\mathbb{C}(2)$ is the algebra of the $2 \times 2$ complex matrices. There is an isomorphism $\mathbb{R}_{1,3}^{0} \simeq \mathbb{R}_{3,0}$ as the reader can easily convince himself. Choose a global orthonormal tetrad coframe $\left\{\gamma^{\mu}\right\}, \gamma^{\mu}=d \mathrm{x}^{\mu}, \mu=0,1,2,3$, and let $\left\{\gamma_{\mu}\right\}$ be the reciprocal tetrad of $\left\{\gamma^{\mu}\right\}$, i.e., $\gamma_{\nu} \cdot \gamma^{\mu}=\delta_{\nu}^{\mu}$. Now, put

$$
\sigma_{i}=\gamma_{i} \gamma_{0}, \mathbf{i}=-\gamma^{0} \gamma^{1} \gamma^{2} \gamma^{3}=-\gamma^{5} .
$$

Observe that $\mathbf{i}$ commutes with bivectors and thus acts like the imaginary unity i $=\sqrt{-1}$ in the even subbundle $\mathcal{C} \ell^{0}(M, g)=\bigcup_{x \in M} \mathcal{C} \ell^{0}\left(T_{x}^{*} M, g_{x}\right) \hookrightarrow$ $\mathcal{C} \ell(M, \mathrm{~g})$, which may be called the Pauli bundle. Now, the electromagnetic field is represented in $\mathcal{C} \ell(M, g)$ by $F=\frac{1}{2} F^{\mu \nu} \gamma_{\mu} \wedge \gamma_{\nu} \in \sec \wedge^{2} T^{*} M \hookrightarrow \sec \mathcal{C} \ell(M, g)$ with

$$
F^{\mu \nu}=\left(\begin{array}{cccc}
0 & -E_{1} & -E_{2} & -E_{3} \\
E_{1} & 0 & -B_{3} & B_{2} \\
E_{2} & B_{3} & 0 & -B_{1} \\
E_{3} & -B_{2} & B_{1} & 0
\end{array}\right),
$$

where $\left(E_{1}, E_{2}, E_{3}\right)$ and $\left(B_{1}, B_{2}, B_{3}\right)$ are the usual Cartesian components of the electric and magnetic fields. Then, as it is easy to verify we can write

$$
F=\mathbf{E}+\mathrm{iB},
$$

with, $\mathbf{E}=\sum_{i=1}^{3} E_{i} \sigma_{i}, \mathbf{B}=\sum_{i=1}^{3} B_{i} \sigma_{i}$. 
Remark 23 Although $\mathbf{E}$ and $\mathbf{B}$ are 2 -form fields in $\mathcal{C} \ell(M, \mathrm{~g})$ they may be identified, once we fix an inertial reference frame (i.e., fix the $\gamma^{0}$ field) with time dependent Euclidean vector fields $\vec{E}, \vec{B}$ and thus we call them "Euclidean vector fields" in $\mathcal{C} \ell^{0}(M, \mathrm{~g})$.

For the electric current density $J_{e}=\rho \gamma^{0}+J^{i} \gamma_{i}$ we can write

$$
\gamma_{0} J_{e}=\rho-\mathbf{j}=\rho-J^{i} \sigma_{i} .
$$

For the Dirac operator we have

$$
\gamma_{0} \boldsymbol{\partial}=\frac{\partial}{\partial x^{0}}+\sum_{i=1}^{3} \sigma_{i} \partial_{i}=\frac{\partial}{\partial t}+\nabla .
$$

Multiplying both members of Eq.(58) on the left by $\gamma_{0}$ we obtain

$$
\begin{aligned}
\gamma_{0} \boldsymbol{\partial} F & =\gamma_{0} J, \\
\left(\frac{\partial}{\partial t}+\nabla\right)(\mathbf{E}+\mathbf{i B}) & =\rho-\mathbf{j}
\end{aligned}
$$

From Eq. (85) we obtain

$$
\partial_{0} \mathbf{E}+\mathbf{i} \partial_{0} \mathbf{B}+\nabla \bullet \mathbf{E}+\nabla \curlywedge \mathbf{E}+\mathbf{i} \nabla \bullet \mathbf{B}+\mathbf{i} \nabla \curlywedge \mathbf{B}=\rho-\mathbf{j} .
$$

In Eq.(86) for any "vector field" $\mathbf{A} \in \mathcal{C} \ell^{0}(M, \mathrm{~g})(\hookrightarrow \mathcal{C} \ell(M, \mathrm{~g}))$,

$$
\begin{aligned}
\nabla \bullet \mathbf{A} & =\sigma_{i} \bullet\left(\partial_{i} \mathbf{A}\right), \\
\nabla \curlywedge \mathbf{A} & =\sigma_{i} \curlywedge\left(\partial_{i} \mathbf{A}\right),
\end{aligned}
$$

with the symbols $\bullet$ being defined through

$$
\begin{aligned}
\sigma_{i} \bullet \sigma_{j} & =\frac{1}{2}\left(\sigma_{i} \sigma_{j}+\sigma_{j} \sigma_{i}\right)=\delta_{i j}, \\
\sigma_{i} \curlywedge \sigma_{j} & =\frac{1}{2}\left(\sigma_{i} \sigma_{j}-\sigma_{j} \sigma_{i}\right) .
\end{aligned}
$$

We define next the vector product of two "vector fields" $\mathbf{C}=\sum_{i=1}^{3} C_{i} \sigma_{i}$ and $\mathbf{D}=\sum_{i=1}^{3} D_{i} \sigma_{i}$ as the dual (see, e.g., [54]) of the "bivector field" $\mathbf{C} \curlywedge \mathbf{D}$ through the formula

$$
\mathbf{C} \times \mathbf{D}=-\mathbf{i}(\mathbf{C} \curlywedge \mathbf{D}) .
$$

Finally, for any "vector field" $\mathbf{A} \in \mathcal{C} \ell^{0}(M, \mathrm{~g})(\hookrightarrow \mathcal{C} \ell(M, \mathrm{~g}))$ we define the rotational operator $\nabla \times$ by

$$
\nabla \times \mathbf{A}=-\mathbf{i}(\nabla \curlywedge \mathbf{A}) .
$$

Using these concepts we obtain from Eq. (866) by equating terms with the same grades (in the Pauli subbundle) 

(a) $\quad \nabla \cdot \mathbf{E}=\rho$,
(c) $\nabla \times \mathbf{E}+\partial_{0} \mathbf{B}=0$,
(b) $\nabla \times \mathbf{B}-\partial_{0} \mathbf{E}=\mathbf{j}$,
(d) $\quad \nabla \cdot \mathbf{B}=0$,

which we recognize as the system of Maxwell equations in the usual vector (engineering) notation. However, the following remark is necessary.

Remark 24 From the above developments we see that a direct formulation of electromagnetism using time dependent fields, which are taken as sections of the Pauli subbundle $\mathcal{C} \ell^{0}(M, \mathrm{~g})$, uses only vector fields, once an orientation (say i) is fixed, thus dispensing the axial vector fields of the traditional Gibbs-Heaviside formulation and the more sophisticated formalism of tensors and tensor densities introduced by [57] and presented as a necessity by some other authors. Moreover, Eq.(89) leaves also clear that the definition of the vector product depends - in each inertial frame (i.e., when we fix field $\gamma^{0}$ ) - on the choice of an orientation in the affine Euclidean rest space $S$ [56] of that frame. It implies that if we change the orientation of $S$, i.e., choose $-\mathbf{i}$ (instead of $\mathbf{i}$ ) in the definition of the vector product, we need to change $\mathbf{B} \mapsto-\mathbf{B}$, which means that the circulation of the magnetic field around a (very long) wire conducting current is conventional 20.

\section{Conclusions}

We showed that in any relativistic spacetime $\left(M, \mathbf{g}, D, \tau_{\mathbf{g}}, \uparrow\right)$, which is necessarily an orientable and time orientable manifold, electromagnetism can be coherently formulated using only pair form fields or pair and impair form fields, contrary to some claims appearing in the literature. The use of pair and impair form fields is necessary only if a non orientable manifold models our universe. However, a manifold of this kind cannot (according to a well known result [21]) represent the spacetime of our universe, where spinor fields live. Moreover we showed that using the Clifford bundle of (pair) forms we can give a formulation of electromagnetism that is compatible with those two formulations using only pair form fields or pair and impair form fields. Each one of those formulations depends only on a mathematical choice that does not seem to imply in any observable consequence.

An eventual objection to our formulation not discussed above, appeared in 25, which claims that the description of electromagnetism using the Clifford bundle formalism is not consistent if magnetic monopoles exist. Now, using that formalism the generalized Maxwell equations read

$$
\partial F=J-\underset{\tau_{g}}{\star} J_{m},
$$

where $J$ is the pair electric current 1-form field and $J_{m}$ is the pair magnetic current 1-form field, and the claim in [25] is that the Clifford bundle formalism implies that $J_{m}=0$ Since this statement appears from time to time it is opportune to recall here that it has been proved wrong in [52, 54, since based on 
a misunderstanding that says that if the electric charges are scalars the magnetic charges must be pseudo-scalars. It also must be said that even if magnetic monopoles do not exist, Eq. (92) is important. The reason is the following. It can be shown that in the Clifford bundle formalism the standard Dirac equation describing, say the interaction of an electron field with the electromagnetic field, is represented by an equation called the the Dirac-Hestenes 29] equation which can be putted in that form

$$
\partial \psi \gamma^{2} \gamma^{1}+m \psi \gamma^{0}+q A \psi=0
$$

where $\psi$ is a Dirac-Hestenes spinor field [39, [53, [54, a mathematical object represented in a given inertial frame $\mathbf{I}=\partial / \partial \mathrm{x}^{0}$ and once a spin-frame is fixed by a non homogeneous even section of the Clifford bundle 42 ,

$$
\psi=S+F+\tau_{g} P \in \sec \left(\bigwedge^{0} T^{*} M+\bigwedge^{2} T^{*} M+\bigwedge^{4} T^{*} M\right) .
$$

It can then easily be shown that the Dirac-Hestenes equation can be written in the form of Eq.(92), where the "electric" and "magnetic" like currents are non linear functionals depending on $S, F$ and $P$ [52, 54].

A last observation is necessary. We are sure that an attentive reader which has not been yet introduced to the Clifford bundle formalism may have become intrigued with our statement in the abstract that pair forms may be used coherently besides in electromagnetism, also in any other physical theory. We just mentioned that Dirac equation can be represented by sum of nonhomogeneous even sections of the Clifford bundle. But, someone may still eventually ask: and what about Einstein's gravitational theory which is formulated with a symmetric metric field as its fundamental field? Well, gravitational theory may also be formulated that field being represented by a set of four linearly independent 1-form fields living on $M$

The details of how this is done can be found in, e.g., [41, 50, 54] and that fact seems to give even more importance to the modern theory of differential forms which started with Cartan.

\section{A How to Calculate $F$}

\section{A.1 Green's Identity for Differential Forms}

In this section $M$ is a $n$-dimensional differentiable manifold and $\mathbf{g} \in \sec T_{0}^{2} M$ is a metric on $M$ of arbitrary signature $(p, q)$, with $p+q=n$. Moreover, we denote by $\mathrm{g} \in \sec T_{2}^{0} M$ the metric on the cotangent bundle such that in an arbitrary coordinate basis where $\mathbf{g}=g_{\mu \nu} d x^{\mu} \otimes d x^{\nu}$ and $\mathrm{g}=g^{\mu \nu} \frac{\partial}{\partial x^{\mu}} \otimes \frac{\partial}{\partial x^{\nu}}$, it is $g_{\mu \nu} g^{\mu \alpha}=$ $\delta_{\nu}^{\alpha}$. We suppose moreover that $\bigwedge T^{*} M$ and $\mathcal{C}(M, \mathrm{~g})$ are respectively the exterior and Clifford algebra bundles of $M$. Let $P \in \sec \bigwedge^{p} T^{*} M \subset \sec \mathcal{C}(M, \mathrm{~g})$. We shall derive an integral identity involving $P, d P$ a $\delta P$ and a Green (extensor)

\footnotetext{
${ }^{42}$ More precisely, Dirac-Hestenes spinor fields are some equivalent classes of even non homogeneous differential forms. See, [39, 53] for details.
} 
distribution $43 \mathbf{G}_{\breve{x}} \in \sec \bigwedge^{p} T^{*} \breve{M} \otimes \sec \bigwedge^{n-p} T^{*} M$ that is a generalization of the well known Green's identities of classical vector calculus. This identity is crucial in order to obtain a formula solving certain differential equations satisfied by $P$.

Let $\left\{\theta^{j}, \theta_{j}\right\}$ be a pair reciprocal bases for $\bigwedge^{1} T^{*} M \hookrightarrow \mathcal{C}(M, \mathrm{~g})$. In what follows the notation $\breve{\theta}_{i}\left(\breve{\theta}^{i}\right)$ means that these forms are calculated at a point $\breve{x} \in \breve{M}$. Now, we introduce the Dirac extensor distribution $\delta_{\breve{x}} \in \sec \bigwedge^{p} T^{*} \breve{M} \otimes$ $\sec \bigwedge^{n-p} T^{*} M$ by

$$
\int \delta_{\breve{x}} \wedge P(x)=P(\breve{x})
$$

where $\delta_{\breve{x}}$ has support only at $\breve{x}$. If $\left\{x^{i}\right\}$ are the coordinate of a chart of an atlas of $M$ and if we choose $\left\{\theta^{j}=d x^{j}, \theta_{j}=g_{i j} d x^{i}\right\}$ then we can easily verify that

$$
\begin{aligned}
\delta_{\breve{x}} & =\frac{(-1)^{p(n-p)}}{p !} \breve{\theta}_{i_{1} \ldots i_{p}} \otimes \star \theta^{i_{1} \ldots i_{p}} \delta(x-\breve{x}), \\
\delta(x-\bar{x}) & =\delta\left(x^{1}-\breve{x}^{1}\right) \ldots \delta\left(x^{n}-\breve{x}^{n}\right), \\
\breve{\theta}_{i_{1} \ldots i_{p}} & =\breve{\theta}_{i_{1}} \wedge \cdots \wedge \breve{\theta}_{i_{p}}, \theta^{i_{1} \ldots i_{p}}=\theta^{i_{1}} \wedge \cdots \wedge \theta^{i_{p}},
\end{aligned}
$$

where in Eq.(96) $\delta\left(x^{i}-\breve{x}^{i}\right), i=1,2, \ldots, n$ are the usual (scalar) Dirac measures.

The Green distribution is supposed to satisfy the following differential equation

$$
\square \mathbf{G}_{\breve{x}}=-(d \delta+\delta d) \mathbf{G}_{\breve{x}}=\delta_{\breve{x}} .
$$

We now prove the following identity:

$$
\begin{aligned}
\delta_{\breve{x}} & \wedge P=(-1)^{n+p}\left[d \mathbf{G}_{\breve{x}} \wedge \delta P-\delta \mathbf{G}_{\breve{x}} \wedge d P\right] \\
& -d\left[\delta \mathbf{G}_{\breve{x}} \wedge P-(-1)^{n p+p+s+1} \underset{\tau_{\mathrm{g}}}{\star} P \wedge \underset{\tau_{\mathrm{g}}}{\star} d \mathbf{G}_{\breve{x}}\right] .
\end{aligned}
$$

We start with the product $d \mathbf{G}_{\breve{x}} \wedge \delta P$ and make some transformations on it using the definition of the Hodge coderivative and some other well known

\footnotetext{
${ }^{43}$ The distribution $\mathbf{G}_{\breve{x}}$ is also called a $p$-form-valued de Rham current. Rigorously we should write $P \in \sec \mathfrak{D}^{\prime}\left(M, \bigwedge^{p} T^{*} M\right) \subset \sec \mathfrak{D}^{\prime}(M, \mathcal{C}(M, \mathrm{~g}))$ and $\mathbf{G}_{\breve{x}} \in \sec \bigwedge^{p} T^{*} \breve{M} \otimes$ $\sec \mathfrak{D}^{\prime}\left(M, \bigwedge^{n-p} T^{*} M\right) \hookrightarrow \sec \bigwedge^{p} T^{*} \bar{M} \otimes \sec D^{\prime}(M, C \ell(M, \mathrm{~g}))$ where $\sec \mathfrak{D}^{\prime}\left(M, \bigwedge^{n-p} T^{*} M\right)$ is the space of the linear functionals over the sections of $\bigwedge^{p} T^{*} M$ of $p$-forms of compact support (in the sense of its action as, e.g., in Eq. (95). $\breve{M}$ is a copy of $M$ and is there to recall that $\mathbf{G}_{\breve{x}}$ is a two point distribution.
} 
identities involving the exterior product 44 . We then have

$$
\begin{aligned}
& d \mathbf{G}_{\breve{x}} \wedge \delta P=(-1)^{n(p+1)+s+1} d \mathbf{G}_{\breve{x}} \wedge \underset{\tau_{g}}{\star d} \underset{\tau_{g}}{\star} P \\
& =(-1)^{n p+n+s+1} d \underset{\tau_{g}}{\star} P \wedge \underset{\tau_{g}}{\star} d \mathbf{G}_{\bar{x}} \\
& \left.=(-1)^{s+1} d \underset{\tau_{\mathbf{g}}}{\star} P \wedge \underset{\tau_{\mathbf{g}}}{\star} d \mathbf{G}_{\breve{x}}\right)-(-1)^{n+p} \delta d \mathbf{G}_{\breve{x}} \wedge P \\
& =(-1)^{s+1} d\left(\underset{\tau_{\mathbf{g}}}{\star} P \wedge \underset{\tau_{\mathbf{g}}}{\star d} \mathbf{G}_{\breve{x}}\right)+(-1)^{n+p}\left[(-\delta d-d \delta) \mathbf{G}_{\breve{x}} \wedge P\right] \\
& +(-1)^{n+p} d \delta \mathbf{G}_{\breve{x}} \wedge P \\
& \left.=(-1)^{s+1} d \underset{\tau_{g}}{\star} P \wedge \underset{\tau_{g}}{\star} d \mathbf{G}_{\breve{x}}\right)+(-1)^{n+p} \delta_{\breve{x}} \wedge P+(-1)^{n+p} d\left(\delta \mathbf{G}_{\breve{x}} \wedge P\right) \\
& +\delta \mathbf{G}_{\breve{x}} \wedge d P,
\end{aligned}
$$

from where Eq.(98) follows.

Integrating both sides on the $n$-dimensional region $\mathcal{U} \subset M$ we have 45

$$
\begin{aligned}
P(\breve{x}) & =(-1)^{n+p} \int_{\mathcal{U}}\left[d \mathbf{G}_{\breve{x}} \wedge \delta P-\delta \mathbf{G}_{\breve{x}} \wedge d P\right] \\
& \left.-\int_{\partial \mathcal{U}} \delta \mathbf{G}_{\breve{x}} \wedge P-(-1)^{n+p+s} \underset{\tau_{g}}{\star} d \mathbf{G}_{\breve{x}} \wedge \underset{\tau_{g}}{\star} P\right] .
\end{aligned}
$$

\section{A.2 Solution of $\partial F=J$}

We now applies the above formula for solving the equation $\partial F=J$ in a Minkowski manifold. We start by choosing a chart with coordinates $\left\{\mathrm{x}^{\mu}\right\}$ in the ELPG. We write as in the text $\gamma^{\mu}=d \mathrm{x}^{\mu}$ and $\gamma_{\mu}=\eta_{\mu \nu} \gamma^{\nu}$. Then, since $\partial F=J$ is equivalent to $d F=0$ and $\delta F=-J$, we have using the retarded solution

$$
\begin{aligned}
\mathbf{G}_{s}(\mathrm{x}-\breve{\mathrm{x}}) & =\frac{1}{2} \breve{\gamma}_{\mu_{1} \mu_{2}} \otimes \underset{\tau_{\mathrm{g}}}{\star} \gamma^{\mu_{1} \mu_{2}} G_{s}(\mathrm{x}-\breve{\mathrm{x}}), \\
\partial^{2} G_{s}(\mathrm{x}-\breve{\mathrm{x}}) & =\delta(\mathrm{x}-\breve{\mathrm{x}})
\end{aligned}
$$

where $G_{s}$ is the scalar Green retarded function (see, e.g., 65]), which vanishes outside the light cone at $x$. Then

$F(\mathrm{x})=-\int_{\mathcal{U}} d \mathbf{G}_{s}(\mathrm{x}-\breve{\mathrm{x}}) \wedge J(\breve{\mathrm{x}})-\int_{\partial \mathcal{U}} \delta \mathbf{G}_{s}(\mathrm{x}-\breve{\mathrm{x}}) \wedge F(\breve{\mathrm{x}})+\underset{\tau_{\mathrm{g}}}{\star} d \mathbf{G}_{s}(\mathrm{x}-\breve{\mathrm{x}}) \wedge \underset{\tau_{\mathrm{g}}}{\star} F(\breve{\mathrm{x}})$

\footnotetext{
${ }^{44}$ See, e.g., Section 2.4 .2 of $[54$.

${ }^{45}$ Analogous equation to Eq. 99] appears in Thirring's book 61. However take care on comparing the equations there and here because of some $(-1)$ signs arising due to different definitions of the Hodge coderivative.
} 
and supposing that $F$ vanishes on the boundary $\partial \mathcal{U}$ we end with

$$
F(\mathrm{x})=-\int_{\mathcal{U}} d \mathbf{G}(\mathrm{x}-\breve{\mathrm{x}}) \wedge J(\breve{\mathrm{x}}) .
$$

This equation shows explicitly that $F \rightarrow-F$ when we decide to relabel the charges entering $J$ from $q^{(i)}$ to $-q^{(i)}$ something that as already discussed in the

text happens if we calculate $\int_{\tau_{g}} J$ in a chart with a different orientation than the positive one defined by $\gamma^{0} \wedge \gamma^{1} \wedge \gamma^{2} \wedge \gamma^{3}$.

\section{References}

[1] Abraham, R., Marsden, J. E., and Ratiu, T., Manifolds, Tensor Analysis, and Applications (2nd. Edition), Springer-Verlag, New York, 1988.

[2] Bott, R. and Tu, L. W., Differential Forms in Algebraic Topology (third edition), Springer, Berlin, 1995.

[3] Benn, I. M. and Tucker, R. W., An Introduction to Spinors and Geometry with Applications in Physics, Adam Hilger, Bristol and New York, 1987.

[4] Cartan, E., Sur Certaínes Expressions Différentielles et le Problème de Pfaff, Ann. Sci. École Norm. Sup. (3 ${ }^{e}$ série) 16, 239-332 (1899). [http://www . numdam.org/item?id=ASENS_1899_3_16_239_0]

[5] Cartan, E., Sur les Varietés à Connexion Affine, et la Théorie de la Relativité Généralisée (premiére partie), Ann. Sci. École Norm. Sup. 41, 1-25 (1924) [http://www.numdam.org/item?id=ASENS_1924_3_41_1_0]

[6] Choquet-Bruhat, Y., DeWitt-Morette, C., and Dillard-Bleick, M., Analysis, Manifolds and Physics (revised edition), North-Holland Publ. Co., Amsterdam, 1982.

[7] Burke, W. L., Applied Differential Geometry, Cambridge University Press, Cambridge, 1985.

[8] van Dantzig, The Fundamental Equations of Electromagnetism Independence of Metric Geometry, Proc. Cambridge Phil. Soc. 30, 421-427 (1934).

[9] Darling, R. W. R., Differential Forms and Connections, Cambridge Univ. Press, Cambridge, 1994.

[10] Delphenich, D. H., On the Axioms of Topological Electromagnetism, Ann. der Physik 14, 347-377 (2005) [arXiv:hep-th/0311256v2].

[11] Deschamps, G., Electromagnetism and Differential Forms, Proc. IEEE 69, 676-896 (1981).

[12] Felsager, B., Geometry, Particles and Fields, Springer, New York, 1998. 
[13] Edelen, D. G. B, A Metric Free Electrodynamics with Electric and Magnetic Charges, Ann. Phys. 112, 366-400 (1978).

[14] Elitzur, A. C., and Shinitzky, M., P-Violation Manifested at the Molecular Level - A Simple Means for an Absolute Definition of "Left" vs. "Right". [arXiv:physics/0601010].

[15] Fernández, V. V., Moya, A. M., and Rodrigues, W. A., Jr., Geometric Algebras and Extensors, Int. J. Geom. Meth. Math. Phys. 4 927-964 (2007) [math.DG/0703090] .

[16] Fernández, V.V., Moya, A. M., and Rodrigues, W. A. Jr., Multivector and Extensor Fields in Smooth Manifolds, Int. J. Geom. Meth. Math. Phys. 4, 965-985 (2007) [arXiv:math/0501559v5 [math.DG]].

[17] Feynman, R. P., Leighton, R. B., and Sands, M., The Feynman Lectures on Physics, vol. II, Addison-Wesley Publ. Co., Inc., Reading, MA, 1964.

[18] Feynman, R. P., The Character of Physical Law, Penguin, New York, 1965.

[19] Flanders, H., Differential Forms with Applications to Physical Sciences, Academic Press, New York, 1963.

[20] Frankel, T., The Geometry of Physics, Cambridge University Press, Cambridge, 1997.

[21] Geroch, R. Spinor Structure of Space-Times in General Relativity I, J. Math. Phys. 9, 1739-1744 (1988).

[22] Göeckler, M., and Scückler, T., Differential Geometry, Gauge Theories and Gravity, Cambridge Univ. Press, Cambridge, 1987.

[23] Graham, G. M. and Lahoz, D. G., Observation of Static Electromagnetic Angular Momentum in Vacua, Nature 285, 154 - 155 (1980).

[24] Grifftihs, D. J., Note on "Field Versus Action-at-a-Distance in a Static Situation by N. L. Sharma, Am. J. Phys. 57, 558-558 (1989).

[25] Gsponer, A., On the "equivalence" of Maxwell and Dirac equations, Int. J. Theor. Phys. 41, 689-694 (2002).

[26] Hehl, F. and Obukhov, Y., Spacetime Metric from Linear Electrodynamics $I, I I$ [arXiv:gr-qc/9904067v2], [arXiv:gr-qc/9911096v1].

[27] Hehl, F. W., Obukhov, Y., Rivera J.-P., and Schmid, H., Magnetoelectric $\mathrm{Cr}_{2} \mathrm{O}_{3}$ and Relativity Theory [arXiv:0903.1261v1 [cond-mat.other]].

[28] Hehl, F. W. and Obukhov, Yu. N., Foundations of Classical Electrodynamics, Charge, Flux and Metric, Birkhäuser, Boston 2003.

[29] Hestenes, D., Spacetime Algebra, Gordon and Breach Sci. Publ., New York, 1966. 
[30] Hehl, F. W. and Obukhov, Y.N., Spacetime Metric from Local and Linear Electrodynamics: A New Axiomatic Scheme, Lect. Notes Phys. 702, 163187 (2006) [arXiv:gr-qc/0508024v1].

[31] Hurley, D. J., and Vandyck, M. A., Geometry, Spinors and Applications, Springer, New York, and Praxis Publ., Chichester, 2000.

[32] Itin, Y. and Friedman, Y., Backwards in Minkowski Road. From 4D to 3D Maxwellian Electromagnetism, Ann. der Physik 17, 769-786 (2008) [arXiv:0807.2625v1 [gr-qc]].

[33] Jackson, J. D., Classical Electrodynamics (second edition), John Wiley \& Sons, Inc., New York, 1975.

[34] Jancewicz, B., The Extended Grassmann Algebra in $\mathbb{R}^{3}$, in Baylis W E (editor), Clifford (Geometric) Algebras with Applications in Physics, Mathematics and Engineering, pp. 389-421, Birkhäuser, Berlin, 1996.

[35] Kiehn, R. M., Charge is a Pseudoscalar [http://www22.pair.com/csdc/pdf/ptpost.pdf].

[36] Kottler, F., Maxwell'sche Gleichungen und Metric, Sitzungsber. Akad. Wien IIa 131, 119-146 (1922).

[37] Lawson, H. B. Jr., Foliations, Bull. Am. Math. Soc. 80, 369-417 (1974).

[38] Marmo, G., Parascoli, E., and Tulczyjew, W. M., Spacetime Orientations and Maxwell Equations, Rept. Math. Phys. 56, 209-248 (2005) [arXiv:0708.3543v1 [math-ph]].

[39] Mosna, R.A. and Rodrigues, W. A . Jr., The Bundles of Algebraic and Dirac-Hestenes Spinor Fields, J. Math. Phys. 45, 2945-2966 (2004) [arXiv:math-ph/0212033v5].

[40] Moya, A. M., Fernández, V. V., and Rodrigues, W. A. Jr., Multivector Functions of a Multivector Variable, Adv. Appl. Clifford Algebras 11, 7991 (2001) [arXiv:math/0212223] .

[41] Notte-Cuello, E., and Rodrigues, W. A. Jr., A Maxwell Like Formulation of Gravitational Theory in Minkowski Spacetime, Int. J. Mod. Phys. D 16, 1027-1041 (2007) [arXiv:math-ph/0608017v5].

[42] Parrott, S., Relativistic Electrodynamics and Differential Geometry, Springer-Verlag, New York, 1987.

[43] Post, E. J., Formal Structure of Electromagnetics, Dover Publ., Inc., New York, 1997.

[44] Post, E. J., The Constitutive Map and Some of its Ramifications, Ann. Phys. 71, 497-518 (1972). 
[45] Post, E. J., Quantum Reprograming, Kluwer Academic Publ., Dordrecht, 1995.

[46] Puntigam, R. A., Lämmerzahl, C., and Hehl, F. W., Maxwell's Theory on a post-Riemannian Spacetime and the Equivalence Principle, Class. Quantum Grav. 14, 1347-1356 (1997).

[47] de Rham, G., Variétés differentiables. Formes, Courants, Formes Harmoniques, Actualités Sci. Ind. 1222, Hermann, Paris 1960.

[48] Riesz, M., Clifford Numbers and Spinors: with Riesz's Private Lectures to E. Folke Bolinder and a Historical Review by Pertti Lounesto, Fundamental Theories of Physics 54, Springer, Berlin, 1993.

[49] da Rocha, R. and Vaz Jr. J, Extended Clifford and Grassmann Algebras, Adv. Applied Clifford Algebras 16, 103-125 (2006) [arXiv:math-ph/0603050v1].

[50] Rodrigues, W. A. Jr., and de Souza, Q. A. G., The Clifford Bundle and the Nature of the Gravitational Field, Found. Phys. 23, 1465-1490 (1993).

[51] Rodrigues, W. A. Jr., de Souza, Q. A. G.,and Bozhkov, Y., The Mathematical Structure of Newtonian Space-Time, Found. Phys. 25, 871-924 (1995).

[52] Rodrigues, W. A. Jr., The Relation between Dirac, Maxwell and SeibergWitten Equations, Int. J. Mathematics and Mathematical Sci. 2003, 27072734 (2003) [arXiv:math-ph/0212034v3].

[53] Rodrigues, W. A. Jr., Algebraic and Dirac-Hestenes Spinors and Spinor Fields, J. Math. Phys. 45, 2908-2994 (2004) [arXiv:math-ph/0212030v6].

[54] Rodrigues, W. A. Jr. and Capelas de Oliveira, E., The Many Faces of Maxwell, Dirac and Einstein Equations. A Clifford Bundle Approach, Lecture Notes in Physics 722, Springer, Heidelberg, 2007.

[55] Salingaros, N., Electromagnetism and the Holomorphic Properties of Spacetime, J. Math. Phys. 22, 1919-1925 (1981).

[56] Sachs, R. K. and Wu, H., General Relativity for Mathematicians, Springer, New York, 1977.

[57] Schönberg, M., Electromagnetism and Gravitation, Brazilian J. Phys. 1, 91-122 (1971).

[58] Schouten, J. A., Tensor Analysis for Physicists (second edition), Dover Publ., Inc., New York, 1989.

[59] Schutz, B., Geometrical Methods of Mathematical Physics, Cambridge Univ. Press, Cambridge, 1980. 
[60] Sharma, N. L., Field Versus Action-at-a-Distance in a Static Situation, Am. J. Phys. 56, 420-558 (1989).

[61] Thirring, W., Classical Field Theory. A Course on Mathematical Physics vol. 2, Springer-Verlag, N. York, 1979.

[62] Tonti, E., Sulla Struttura Formale delle Teorie Fisiche, Rendiconti del Seminario Matematico e Fisico di Milano XLVI, 163-257 (1976).

[63] Tonti, E., On the Geometrical Structure of Electromagnetism, in Ferrarese G. (editor), Gravitation, Electromagnetism and Geometrical Structures, pp. 281-308, Pitagora Editirice, Bologna, 1996.

[64] Truesdell, C. and Toupin, R., The Classical Field Theories, in Függe, S. (ed.), Handbuch der Physik Physics vol. III/I, 226-793, Springer-Verlag, Berlin, 1960.

[65] Vladimirov, V. S., Equations of Mathematical Physics, Marcel Decker Inc., New York, 1971.

[66] Weyl, H., Space Time Matter, Dover Publ., New York, 1952.

[67] Wu, C. S., Ambler, E., Hayward, R., W., Hoppes, D. D., and Hudson, R. P., Experimental Test of Parity Conservation in Beta Decay, Phys. Rev. 105, 1413-1415 (1957). 